\title{
Tree-level splitting amplitudes for a gluon into four collinear partons
}

\author{
Vittorio Del Duca, ${ }^{a, 1}$ Claude Duhr, ${ }^{b}$ Rayan Haindl, ${ }^{a}$ Achilleas Lazopoulos ${ }^{a}$ and \\ Martin Michel ${ }^{c}$ \\ ${ }^{a}$ Institute for Theoretical Physics, ETH Zürich, \\ 8093 Zürich, Switzerland \\ ${ }^{b}$ Theoretical Physics Department, CERN, \\ CH-1211 Geneva 23, Switzerland \\ ${ }^{c}$ Center for Cosmology, Particle Physics and Phenomenology (CP3), UCLouvain, \\ B-1348, Louvain-La-Neuve, Belgium \\ E-mail: delducav@itp.phys.ethz.ch, claude.duhr@cern.ch, \\ haindlr@phys .ethz.ch, lazopoli@phys .ethz.ch, \\ martin.michel@uclouvain. be
}

ABSTRACT: We compute in conventional dimensional regularisation the tree-level splitting amplitudes for a gluon parent which splits into four collinear partons. This is part of the universal infrared behaviour of the QCD scattering amplitudes at next-to-next-to-next-toleading order $\left(\mathrm{N}^{3} \mathrm{LO}\right)$ in the strong coupling constant. Combined with our earlier results for a quark parent, this completes the set of tree-level splitting amplitudes required at this order. We also study iterated collinear limits where a subset of the four collinear partons become themselves collinear.

Keywords: QCD Phenomenology

ARXiv EPrint: 2007.05345

\footnotetext{
${ }^{1}$ On leave from INFN, Laboratori Nazionali di Frascati, Italy.
} 


\section{Contents}

1 Introduction 1

2 Multiple collinear limits $\quad 3$

3 Gluon-initiated splitting amplitudes $\quad 5$

4 Nested collinear limits $\quad 9$

5 Conclusions 13

$\begin{array}{ll}\text { A The iterated collinear limit } & 14\end{array}$

B Tensor structure of gluon parent and sub-parent splitting tensors $\quad 15$

$\begin{array}{ll}\text { C The three-parton splitting tensors } & 17\end{array}$

\section{Introduction}

With the discovery of the Higgs boson by the Large Hadron Collider (LHC) at CERN [1, 2], the Standard Model (SM) of particle physics is a complete theory without any free parameters. Current and future collider experiments will be able to test the SM with an increasing level of precision. The requested precision poses a severe challenge to theory and calls for the development of improved techniques for theoretical predictions accurate at the percent level. One of the main approaches to theoretical collider phenomenology is perturbation theory, in which observables are expanded in the small coupling constants of the theory. Here we focus on QCD observables, and consequently we will be concerned with the computation of higher orders in the strong coupling constant $\alpha_{s}$ of QCD.

Often, leading order predictions in QCD are not reliable. For example it is known that in the case of Higgs production the next-to-leading order corrections almost double the value of the cross section [3, 4]. The next-to-next-to-leading order (NNLO) corrections (in the limit where the top-quark is infinitely heavy) further increase the cross section, and it is only after the inclusion of the next-to-next-to-next-to-leading order $\left(\mathrm{N}^{3} \mathrm{LO}\right)$ corrections that a reliable estimate of the cross section with a residual uncertainty of only a few percent is obtained [5-7]. This example illustrates that reliable QCD predictions at the percent level can most likely only be achieved after the inclusion of corrections at NNLO and beyond. While over the last years a lot of progress was made in NNLO computations, so far, only very few hadron collider processes are known at $\mathrm{N}^{3} \mathrm{LO}$. Inclusive $\mathrm{N}^{3} \mathrm{LO}$ cross sections are known for Higgs in gluon fusion [5-7], bottom-quark fusion [8, 9] and vector boson fusion in the DIS approach [10,11], as well as double Higgs production [12, 13] and Drell-Yan 
production (via the intermediate of an off-shell photon) [14]. At the differential level, only the Higgs rapidity and transverse momentum distributions in vector boson fusion in the DIS approximation [10, 15, 16] are known.

One of the reasons progress towards more results at $\mathrm{N}^{3} \mathrm{LO}$ is difficult lies in the fact that an observable at $\mathrm{N}^{k} \mathrm{LO}$ receives contributions from processes with up to $k$ additional partons in the final state. Each such contribution is individually infrared divergent, with divergences arising in particular from the integration over regions of phase space where the emitted partons are either soft or collinear. Several techniques have been developed at NNLO to compute the relevant phase space integrals with generic acceptance cuts [17-50]. With some abuse of language, we refer to these techniques in the following collectively as subtraction methods. Developing subtracting methods beyond NNLO will be an important step towards obtaining more predictions with percent accuracy. ${ }^{1}$

An important ingredient in the development of subtraction methods is the universal behaviour of the QCD scattering amplitudes in the infrared limits, embodied in universal soft and collinear currents. At NNLO these currents have been computed more than a decade ago, and they include splitting amplitudes for three partons at tree-level and two partons at one-loop, as well as soft currents for the emission of two soft partons at tree-level and one soft parton at one-loop [52-60]. Over the last few years, results have also become available for the universal currents at $\mathrm{N}^{3} \mathrm{LO}$. The two-loop currents for the emission of two collinear or one soft parton were obtained in refs. [61-66], and the one-loop current for the emission of three collinear partons is given in refs. $[67,68] .^{2}$ The tree-level currents include the soft current for the emission of three soft partons [70] and the splitting amplitudes for four collinear partons. The latter have been obtained at the amplitude-level in four dimensions in refs. [55, 71, 72]. Recently, we have published the splitting amplitudes for the squared matrix element in dimensional regularisation, in the case where the parent parton is a quark [73]. The main purpose of this paper is to complete the set of tree-level splitting amplitudes at $\mathrm{N}^{3} \mathrm{LO}$ by providing analytic results in dimensional regularisation for a gluon splitting into four partons.

This paper is organised as follows: in section 2, we review the collinear limit of treelevel amplitudes and give a precise definition of the quantities that we want to compute. In section 3, we present the main result of our paper, namely the computation of the tree-level splitting amplitudes for a gluon parent to split into four collinear partons. The explicit results are too long to be recorded in this paper and are made available in computerreadable form [74]. In section 4 we study the collinear limit of the splitting amplitudes themselves, and we define new universal objects which appear in these iterated limits. We include several appendices with technical material omitted throughout the main text.

\footnotetext{
${ }^{1}$ Some of these methods have already been successfully applied at $\mathrm{N}^{3} \mathrm{LO}$ for processes with a simple final state structure $[10,11,15,51]$.

${ }^{2}$ One-loop currents with three collinear partons are also known for mixed QCD+QED cases [69].
} 


\section{Multiple collinear limits}

We examine the behaviour of tree-level QCD amplitudes in the limit where a given number of massless partons become collinear. Namely, we consider the scattering of $n$ massless particles with momenta $p_{i}$ and with flavour, spin and colour quantum numbers $f_{i}, s_{i}$ and $c_{i}$, respectively, and we analyse the behaviour of the amplitude as $m$ partons of momenta $p_{1}, \ldots, p_{m}$ become simultaneously collinear to some light-like direction $\widetilde{P}$. In this limit, the leading behaviour is described by the amplitude for the production of a massless particle of momentum $\widetilde{P}$ from a scattering of the particles that do not take part in the collinear limit, multiplied by a universal factor, termed the splitting amplitude, which depends only on the $m$ partons in the collinear set.

In order to parametrise the approach to the collinear limit, we introduce a light-cone decomposition for all the momenta in the $m$-parton collinear set,

$$
p_{i}^{\mu}=x_{i} \widetilde{P}^{\mu}+k_{\perp i}^{\mu}-\frac{k_{\perp i}^{2}}{2 x_{i}} \frac{n^{\mu}}{\widetilde{P} \cdot n}, \quad i=1, \ldots, m
$$

where the light-like momentum $\widetilde{P}$ specifies the collinear direction, $\widetilde{P} \cdot k_{\perp i}=0, x_{i}$ are the longitudinal momentum fractions with respect to the parent momentum $P^{\mu}=\sum_{\widetilde{P}_{i=1}}^{m} p_{i}^{\mu}$ and $n^{\mu}$ is an auxiliary light-like vector such that $n \cdot k_{\perp i}=0$ and $n \cdot p_{i} \neq 0 \neq n \cdot \widetilde{P}$, and which specifies how the collinear direction is approached. The collinear limit is then defined as the limit in which the transverse momenta $k_{\perp i}$ approach zero at the same rate. This definition of the collinear limit is frame-independent, and it only depends on the collinear direction $\widetilde{P}$ and the transverse momenta $k_{\perp i}$. In particular it is independent of the choice of the auxiliary vector $n$.

The variables that appear in eq. (2.1) are unconstrained apart from on-shellness and transversality, $n \cdot k_{\perp i}=\widetilde{P} \cdot k_{\perp i}=0$, and so the sums of the momentum fractions $x_{i}$ and the transverse momenta $k_{\perp i}$ are unconstrained. However, the collinear limit is invariant under longitudinal boosts in the direction of the parent momentum $P=\sum_{i=1}^{m} p_{i}$. We trade $x_{i}$ and $k_{\perp i}$ for new quantities $z_{i}$ and $\tilde{k}_{\perp i}$ that are boost-invariant in the direction of the parent momentum. In refs. [54, 73], it was shown that a convenient set of such variables is given by

$$
z_{i}=\frac{x_{i}}{\sum_{j=1}^{m} x_{j}}=\frac{p_{i} \cdot n}{P \cdot n}, \quad \tilde{k}_{\perp i}^{\mu}=k_{\perp i}^{\mu}-z_{i} \sum_{j=1}^{m} k_{\perp j}^{\mu}, \quad i=1, \ldots, m .
$$

It is easy to see that these new variables satisfy the constraints,

$$
\sum_{i=1}^{m} z_{i}=1 \text { and } \sum_{i=1}^{m} \tilde{k}_{\perp i}^{\mu}=0 .
$$

From now on, we only work with these variables, and in order to avoid cluttering notation, we shall drop the tilde on the transverse momenta.

In the limit where a subset of massless particles is collinear, a scattering amplitude factorises as $[75-77]$

$$
\begin{aligned}
\mathscr{C}_{1 \ldots m} \mathcal{M}_{f_{1} \ldots f_{n}}^{c_{1} \ldots c_{n} ; s_{1} \ldots s_{n}}\left(p_{1}, \ldots, p_{n}\right) & \\
& =\mathbf{S p}_{f_{f_{1} \ldots f_{m}}^{c, c_{1} \ldots c_{m} ; s, s_{1} \ldots s_{m}}}^{c} \mathcal{M}_{f f_{m+1} \ldots f_{n}}^{c, c_{m+1} \ldots c_{n} ; s, s_{m+1} \ldots s_{n}}\left(\widetilde{P}, p_{m+1}, \ldots, p_{n}\right),
\end{aligned}
$$


where $\mathscr{C}_{1 \ldots m}$ indicates that the equality holds up to terms that are power-suppressed in the collinear limit, while $f, s$ and $c$ respectively denote the flavour, spin and colour indices of the parent particle. The quantity $\mathbf{S p}$ appearing on the right-hand side is the splitting amplitude, which depends only on the kinematics and the quantum numbers in the collinear set.

For an amplitude whose collinear massless particles occur all in the final state, the factorisation in eq. (2.4) is valid to all orders in perturbation theory. ${ }^{3}$ Accordingly, also the squared matrix element factorises,

$$
\left|\mathcal{M}_{f_{1} \ldots f_{n}}\left(p_{1}, \ldots, p_{n}\right)\right|^{2} \equiv \sum_{\substack{\left(s_{1}, \ldots, s_{n}\right) \\\left(c_{1}, \ldots, c_{n}\right)}}\left|\mathcal{M}_{f_{1} \ldots f_{n}}^{c_{1} \ldots c_{n} ; s_{1} \ldots s_{n}}\left(p_{1}, \ldots, p_{n}\right)\right|^{2}
$$

where in the short-hand notation of the left-hand side the sum over all spin and colour indices of the matrix element is understood. The factorisation of the squared matrix element can be written as

$$
\mathscr{C}_{1 \ldots m}\left|\mathcal{M}_{f_{1} \ldots f_{n}}\left(p_{1}, \ldots, p_{n}\right)\right|^{2}=\left(\frac{2 \mu^{2 \epsilon} g_{s}^{2}}{s_{1 \ldots m}}\right)^{m-1} \hat{P}_{f_{1} \ldots f_{m}}^{s s^{\prime}} \mathcal{T}_{f f_{m+1} \ldots f_{n}}^{s s^{\prime}}\left(\widetilde{P}, p_{m+1}, \ldots, p_{n}\right),
$$

where $g_{s}$ is the strong coupling constant and $\mu$ is the scale introduced by dimensional regularisation, and a sum over repeated indices, in this case the spin indices $s$ and $s^{\prime}$, is implicit, and we introduced the Mandelstam invariant,

$$
s_{1 \ldots m} \equiv\left(p_{1}+\ldots+p_{m}\right)^{2} .
$$

$\mathcal{T}_{f f_{m+1} \ldots f_{n}}^{s s^{\prime}}$ denotes the helicity tensor obtained by not summing over the spin indices of the parent parton,

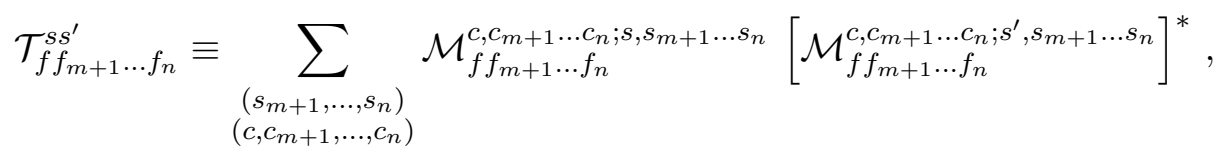

where for brevity we have suppressed the momenta on which the amplitude depends. The tensorial structure of the factorisation in eq. (2.6) is necessary to correctly capture all spin correlations. Due to colour conservation in the hard amplitude there are no nontrivial colour correlations, and we therefore sum over the colour $c$ of the parent parton in eq. (2.8). The quantity $\hat{P}_{f_{1} \ldots f_{m}}^{s f^{\prime}}$ in eq. (2.6) is the (polarised) splitting amplitude for the squared matrix element, which is related to $\mathbf{S p}$ by

$$
\left(\frac{2 \mu^{2 \epsilon} g_{s}^{2}}{s_{1 \ldots m}}\right)^{m-1} \hat{P}_{f_{1} \ldots f_{m}}^{s s^{\prime}}=\frac{1}{\mathcal{C}_{f}} \sum_{\substack{\left(s_{1}, \ldots, s_{m}\right) \\\left(c, c_{1}, \ldots, c_{m}\right)}} \mathbf{S p}_{f f_{1} \ldots f_{m}}^{c, c_{1} \ldots c_{m} ; s, s_{1} \ldots s_{m}}\left[\mathbf{S p}_{f f_{1} \ldots f_{m}}^{c, c_{1} \ldots c_{m} ; s^{\prime}, s_{1} \ldots s_{m}}\right]^{*}
$$

where $\mathcal{C}_{f}$ is the number of colour degrees of freedom of the parent parton with flavour $f$, i.e., $\mathcal{C}_{g}=N_{c}^{2}-1$ for a gluon and $\mathcal{C}_{q}=N_{c}$ for a quark. In eqs. (2.6), (2.9) and henceforth,

\footnotetext{
${ }^{3}$ When the subset of collinear particles contains also initial-state particles, the factorisation in eq. (2.4) is valid in general only for tree amplitudes [78].
} 
the dependence of the splitting amplitude on the transverse momenta $\tilde{k}_{\perp i}$ and momentum fractions $z_{i}$ of the particles in the collinear set is understood. Further, in QCD the flavour of the parent is uniquely determined by the flavours of the particles in the collinear set, thus we suppress the dependence of the splitting amplitude on the left-hand-side of eq. (2.9) on the flavour of the parent parton.

Splitting amplitudes for the squared matrix element have been computed at tree level for the emission of up to three collinear partons in refs. [53, 54], and for the emission of four collinear partons out of a parent quark in ref. [73]. The goal of this paper is to compute the tree-level splitting amplitudes for the squared matrix element for the emission of up to four partons out of a parent gluon, thus completing the set of splitting amplitudes for the emission of up to four collinear partons in QCD. ${ }^{4}$

\section{Gluon-initiated splitting amplitudes}

In this section we present the computation of the gluon-initiated tree-level splitting amplitudes for $m=4$ collinear partons, ${ }^{5}$ which is the main result of our paper. The computation follows the same lines as that for $m=3$ collinear partons in ref. [54]. Our results for the splitting amplitudes are too lengthy to be presented in printed form, but we make them available in computer-readable form [74].

In order to compute an $m$-parton splitting amplitude, we start from an on-shell amplitude for $n=m+3$ partons and take $m$ of them collinear. We perform a uniform rescaling of the transverse momenta $k_{\perp i}$ in eq. (2.1) by a small parameter $\lambda$,

$$
k_{\perp i} \rightarrow \lambda k_{\perp i}, \quad 1 \leq i \leq m .
$$

This ensures that in the collinear limit $\lambda \rightarrow 0$ the $k_{\perp i}$ approach zero at the same rate. We then expand the matrix element into a Laurent series around $\lambda=0$. The leading term corresponds to the coefficient of $1 / \lambda^{2(m-1)}$, which is universal and is described by the collinear factorisation in eq. (2.6).

While the final result of this operation is of course gauge independent, the set of Feynman diagrams that contribute to the leading behaviour in $\lambda$ depend on the gauge choice. We would therefore like to choose a gauge that simplifies the computation as much as possible, e.g., by minimising the number of (interfering) Feynman diagrams that contribute in the collinear limit. In ref. [54] it was argued that it is convenient to work in a physical gauge (e.g., axial gauge), because contributions from Feynman diagrams where collinear partons are separated by a hard propagator are subleading in the collinear limit. Here we work in axial gauge, where the gluon field is subject to the following conditions,

$$
\partial_{\mu} A^{\mu}=n_{\mu} A^{\mu}=0
$$

\footnotetext{
${ }^{4}$ As in ref. [73], we refer to both $\mathbf{S p}$ and $\hat{P}$ simply as splitting amplitudes.

${ }^{5}$ The constraints in eq. (2.3) have not been imposed on our results. This may allow us, through crossing symmetry, to readily obtain the splitting amplitudes for initial-state collinear emissions [79].
} 
where $n$ is an arbitrary light-like reference vector. In this gauge, the gluon propagator takes the form,

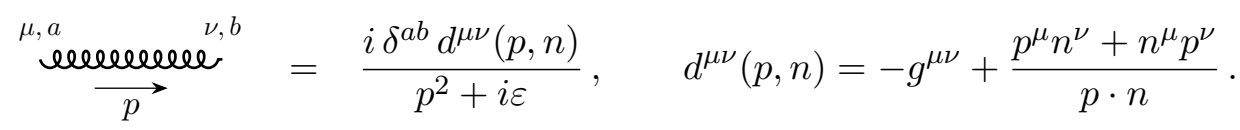

For $p^{2}=0$, the polarisation tensor $d^{\mu \nu}(p, n)$ can be interpreted in three different ways: first, it is the projector onto the $(D-2)$-dimensional space transverse to $p$ and $n$. Second, it is the metric tensor induced by the $D$-dimensional Minkowski metric on that space. Finally, it is the sum of all physical polarisations of a gluon with momentum $p$,

$$
\sum_{s} \varepsilon_{s}^{\mu}(p, n) \varepsilon_{s}^{\nu}(p, n)=d^{\mu \nu}(p, n)
$$

where $\varepsilon_{s}^{\mu}(p, n)$ is the polarisation vector for a gluon with momentum $p$ and transverse polarisation $s=1, \ldots,(D-2)$. In principle, we may choose a different reference vector for each gluon, as long as it is not orthogonal to the momentum. In our case, it is convenient to choose all gauge reference vectors to coincide with the reference vector $n$ appearing in the definition of the collinear limit in eq. (2.1). We can then write the collinear factorisation for a parent gluon in terms of Lorentz indices rather than helicities [54],

$$
\mathscr{C}_{1 \ldots m}\left|\mathcal{M}_{f_{1} \ldots f_{n}}\left(p_{1}, \ldots, p_{n}\right)\right|^{2}=\left(\frac{2 \mu^{2 \epsilon} g_{s}^{2}}{s_{1 \ldots m}}\right)^{m-1} \hat{P}_{f_{1} \ldots f_{m}}^{\mu \nu} \mathcal{T}_{f f_{m+1} \ldots f_{n}, \mu \nu}\left(\widetilde{P}, p_{m+1}, \ldots, p_{n}\right),
$$

where quantities with open Lorentz indices are obtained by amputating the polarisation vectors and inserting the polarisation sum in eq. (3.4). Note that in passing from eq. (2.6) to eq. (3.5) we have implicitly used gauge invariance to eliminate the gauge dependent terms in eq. (3.4). Indeed, since physical polarisation states are transverse, only the transverse part of a Lorentz tensor carries physical information. This is because the non-transverse part vanishes upon contraction with a physical polarisation vector. As a consequence, the helicity tensor $\mathcal{T}_{f f_{m+1} \ldots f_{n}}^{\mu \nu}$ in eq. (3.5) can be chosen to satisfy the transversality condition,

$$
\widetilde{P}_{\mu} \mathcal{T}_{f f_{m+1} \ldots f_{n}}^{\mu \nu}=\widetilde{P}_{\nu} \mathcal{T}_{f f_{m+1} \ldots f_{n}}^{\mu \nu}=0
$$

With this choice, the complete tensor structure of the splitting amplitude contains terms involving the transverse momenta of the collinear partons [54],

$$
\hat{P}_{f_{1} \ldots f_{m}}^{\mu \nu}=g^{\mu \nu} A_{f_{1} \ldots f_{m}}^{(g)}+\sum_{i, j=1}^{m} \frac{\tilde{k}_{\perp i}^{\mu} \tilde{k}_{\perp j}^{\nu}}{s_{1 \ldots m}} B_{i j, f_{1} \ldots f_{m}}^{(g)} .
$$

We stress, however, that the splitting amplitude defined in this way does not vanish upon contraction with $\widetilde{P}$.

Since we work in axial gauge, we do not consider the subset of Feynman diagrams where collinear partons are separated by a hard propagator. The sum of all relevant diagrams 
can be cast in the form,

$$
\begin{aligned}
\mathscr{C}_{1 \ldots m}\left|\mathcal{M}_{f_{1} \ldots f_{n}}\left(p_{1}, \ldots, p_{n}\right)\right|^{2}= \\
\quad=\mathscr{C}_{1 \ldots m}\left[\left(\frac{2 \mu^{2 \epsilon} g_{s}^{2}}{s_{1 \ldots m}}\right)^{m-1}\left[\mathcal{M}_{f f_{m+1} \ldots f_{n}}^{(n) s}\right]^{*} V_{f_{1} \ldots f_{m}}^{(n) s s^{\prime}}\left(p_{1}, \ldots, p_{m}\right) \mathcal{M}_{f f_{m+1} \ldots f_{n}}^{(n) s^{\prime}}\right],
\end{aligned}
$$

where a sum over the spin indices $s, s^{\prime}$ of the intermediate state is understood, and we suppress all colour and spin indices of the external partons. Here $\mathcal{M}_{f f_{m+1} \ldots f_{n}}^{(n) s} \equiv$ $\mathcal{M}_{f f_{m+1} \ldots f_{n}}^{(n) s}\left(P, p_{m+1}, \ldots, p_{n}\right)$ denotes the sum of all Feynman diagrams with an off-shell leg with momentum $P$, flavour $f$ and spin $s$. Note that this subset of Feynman diagrams is by itself not gauge invariant, and the superscript $(n)$ indicates the dependence on the reference vector. The squared off-shell current $V_{f_{1} \ldots f_{m}}^{(n) s s^{\prime}}$ may be written as the interference of two colour-dressed off-shell currents,

$$
\left(\frac{2 \mu^{2 \epsilon} g_{s}^{2}}{s_{1 \ldots m}}\right)^{m-1} V_{f_{1} \ldots f_{m}}^{(n) s s^{\prime}}\left(p_{1}, \ldots, p_{m}\right)=\frac{1}{\mathcal{C}_{f}} \sum_{\substack{\left(s_{1}, \ldots, s_{m}\right) \\\left(c, c_{1}, \ldots, c_{m}\right)}}\left[\mathrm{J}_{f_{1} \ldots f_{m}}^{c, c_{1} \ldots c_{m} ; s^{\prime} s_{1} \ldots s_{m}}\right]^{*} \mathrm{~J}_{f_{1} \ldots f_{m}}^{c, c_{1} \ldots c_{m} ; s s_{1} \ldots s_{m}}
$$

where $\mathcal{C}_{f}$ is defined after eq. (2.9). Note that also $V_{f_{1} \ldots f_{m}}^{(n) s s^{\prime}}$ depends on the gauge vector $n$ before the collinear limit is taken. Since the collinear limit is gauge invariant, this dependence disappears in the limit, and the squared off-shell current reduces to the splitting amplitude,

$$
\mathscr{C}_{1 \ldots m} V_{f_{1} \ldots f_{m}}^{(n) s s^{\prime}}\left(p_{1}, \ldots, p_{m}\right)=\hat{P}_{f_{1} \ldots f_{m}}^{s s^{\prime}} .
$$

We have computed all gluon-initiated splitting amplitudes up to $m=4$, and we reproduce all known results for the cases $m=2$ and 3. The results for $m=4$ are new and are presented for the first time in this paper. There are four different gluon-initiated splitting amplitudes,

$$
g \rightarrow \bar{q}^{\prime} q^{\prime} \bar{q} q, \quad g \rightarrow \bar{q} q \bar{q} q, \quad g \rightarrow \bar{q} g g q, \quad g \rightarrow g g g g .
$$

In the remainder of this section we discuss in more detail the computation of these splitting amplitudes. The explicit results are available in computer-readable form [74].

Let us start by discussing the simplest splitting process, the collinear decay $g \rightarrow \bar{q}^{\prime} q^{\prime} \bar{q} q$ with different quark flavours. There are five diagrams that contribute to the off-shell current $\mathrm{J}_{\bar{q}^{\prime} q^{\prime} \bar{q} q}^{\mu}$ in eq. (3.9). The diagrams are shown in figure 1. Going through the steps outlined above, we find that the result for the splitting amplitude $g \rightarrow \bar{q}^{\prime} q^{\prime} \bar{q} q$ can be decomposed into an 'abelian' and a 'non-abelian' part,

$$
\hat{P}_{\bar{q}_{1}^{\prime} q_{2}^{\prime} \bar{q}_{3} q_{4}}^{\mu \nu}=\frac{1}{4} C_{F} \hat{P}_{\bar{q}_{1}^{\prime} q_{2}^{\prime} \bar{q}_{3} q_{4}}^{\mu \nu(\mathrm{ab})}+\frac{1}{4} C_{A} \hat{P}_{\bar{q}_{1}^{\prime} q_{2}^{\prime} \bar{q}_{3} q_{4}}^{\mu \nu(\mathrm{nab})},
$$

where the indices carried by the parton label refer to the indices of the momenta and the momentum fractions of the partons, and $C_{F}$ and $C_{A}$ denote the quadratic Casimirs of the fundamental and adjoint representations of $\mathrm{SU}(N)$,

$$
C_{F}=\frac{N^{2}-1}{2 N}, \quad C_{A}=N .
$$




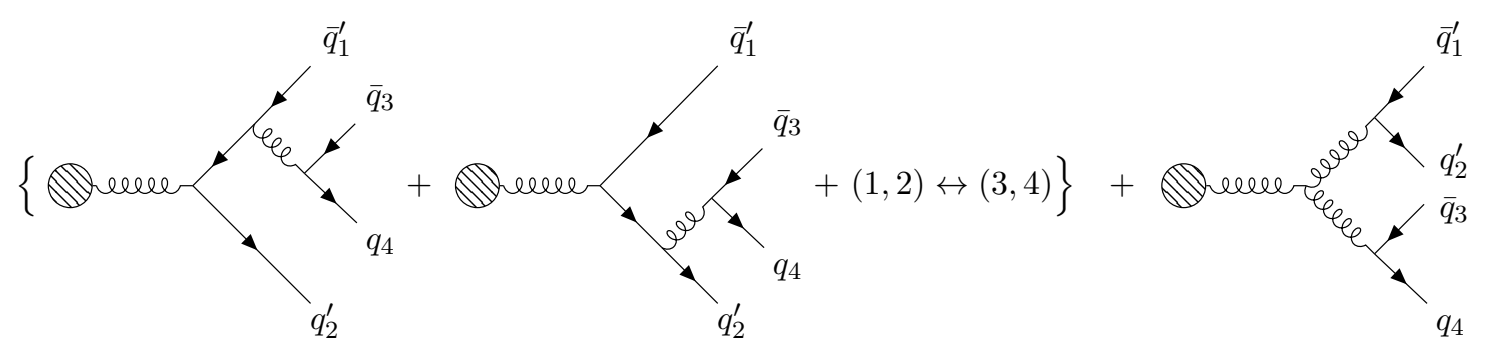

Figure 1. The diagrams contributing to the off-shell current $g \rightarrow \bar{q}_{1}^{\prime} q_{2}^{\prime} \bar{q}_{3} q_{4}$. In the case of identical quarks we also need to include diagrams where the anti-quarks $q_{1}$ and $q_{3}$ are exchanged.

The splitting process $g \rightarrow \bar{q} q \bar{q} q$, in which the final state quarks have the same flavour, $q^{\prime}=q$, includes diagrams where the anti-quarks 1 and 3 are exchanged (or equivalently, where the quarks 2 and 4 are exchanged). This naturally leads to the following representation of the splitting amplitude,

$$
\hat{P}_{\bar{q}_{1} q_{2} \bar{q}_{3} q_{4}}^{\mu \nu}=\left[\hat{P}_{\bar{q}_{1}^{\prime} q_{2}^{\prime} \bar{q}_{3} q_{4}}^{\mu \nu}+(1 \leftrightarrow 3)\right]+\hat{P}_{\bar{q}_{1} q_{2} \bar{q}_{3} q_{4}}^{\mu \nu(\mathrm{id})} .
$$

The term in square brackets contains the splitting amplitude in eq. (3.12) in the case of different flavours and the exchange contributions obtained by permuting the external quarks. The last term in eq. (3.14) is new and captures interference contributions from identical quarks. It is again convenient to display the result in terms of colour factors,

$$
\hat{P}_{\bar{q}_{1} q_{2} \bar{q}_{3} q_{4}}^{\mu \nu(\mathrm{i})}=\frac{1}{2} C_{F}\left(C_{A}-2 C_{F}\right) \hat{P}_{\bar{q}_{1} q_{2} \bar{q}_{3} q_{4}}^{\mu \nu(\mathrm{id})_{1}}+\frac{1}{2} C_{A}\left(C_{A}-2 C_{F}\right) \hat{P}_{\bar{q}_{1} q_{2} \bar{q}_{3} q_{4}}^{\mu \nu(\mathrm{id})_{2}} .
$$

Since $C_{A}-2 C_{F}=\frac{1}{N}$, the interference contributions are colour suppressed.

Next, let us discuss the splitting amplitude $g \rightarrow \bar{q} q g g$. The Feynman diagrams contributing to the off-shell current $\mathrm{J}_{\bar{q} q g g}^{\mu}$ are shown in figure 2 . As usual, we can decompose the splitting amplitude into contributions from different colour factors as follows,

$$
\hat{P}_{\bar{q}_{1} g_{2} g_{3} q_{4}}^{\mu \nu}=\frac{1}{2} C_{F}^{2} \hat{P}_{\bar{q}_{1} g_{2} g_{3} q_{4}}^{\mu \nu(\mathrm{ab})}+\frac{1}{2} C_{A}^{2} \hat{P}_{\bar{q}_{1} g_{2} g_{3} q_{4}}^{\mu \nu(\mathrm{nab})_{1}}+\frac{1}{2} C_{A} C_{F} \hat{P}_{\bar{q}_{1} g_{2} g_{3} q_{4}}^{\mu \nu} .
$$

It is possible to express each colour coefficient in eq. (3.16) in a reduced form by exploiting the symmetry under the exchange of the two external partons,

$$
\hat{P}_{\bar{q}_{1} g_{2} g_{3} q_{4}}^{\mu \nu(X)}=\left(\hat{P}_{\bar{q}_{1} g_{2} g_{3} q_{4}}^{\mu \nu(X) \text { symm. }}+(1 \leftrightarrow 4)\right)+(2 \leftrightarrow 3),
$$

where $(X) \in\left\{(\mathrm{ab}),(\text { nab })_{1},(\text { nab })_{2}\right\}$.

Finally, let us discuss the pure gluon splitting process $g \rightarrow g g g g$, which poses a challenge due to the large degree of Bose symmetry under the exchange of the external gluons. The diagrams contributing to the decay are shown in figure 3. An important step in the computation of the splitting amplitude was to take into account symmetries between different permutatons of the four external gluons in order to minimise the number of terms. We can write $\hat{P}_{g g g g}^{\mu \nu}$ in a symmetrised form as

$$
\hat{P}_{g_{1} g_{2} g_{3} g_{4}}^{\mu \nu}=\hat{P}_{g_{1} g_{2} g_{3} g_{4}}^{\mu \nu}+\left(11 \text { permutations of } g_{1} g_{2} g_{3} g_{4}\right) .
$$



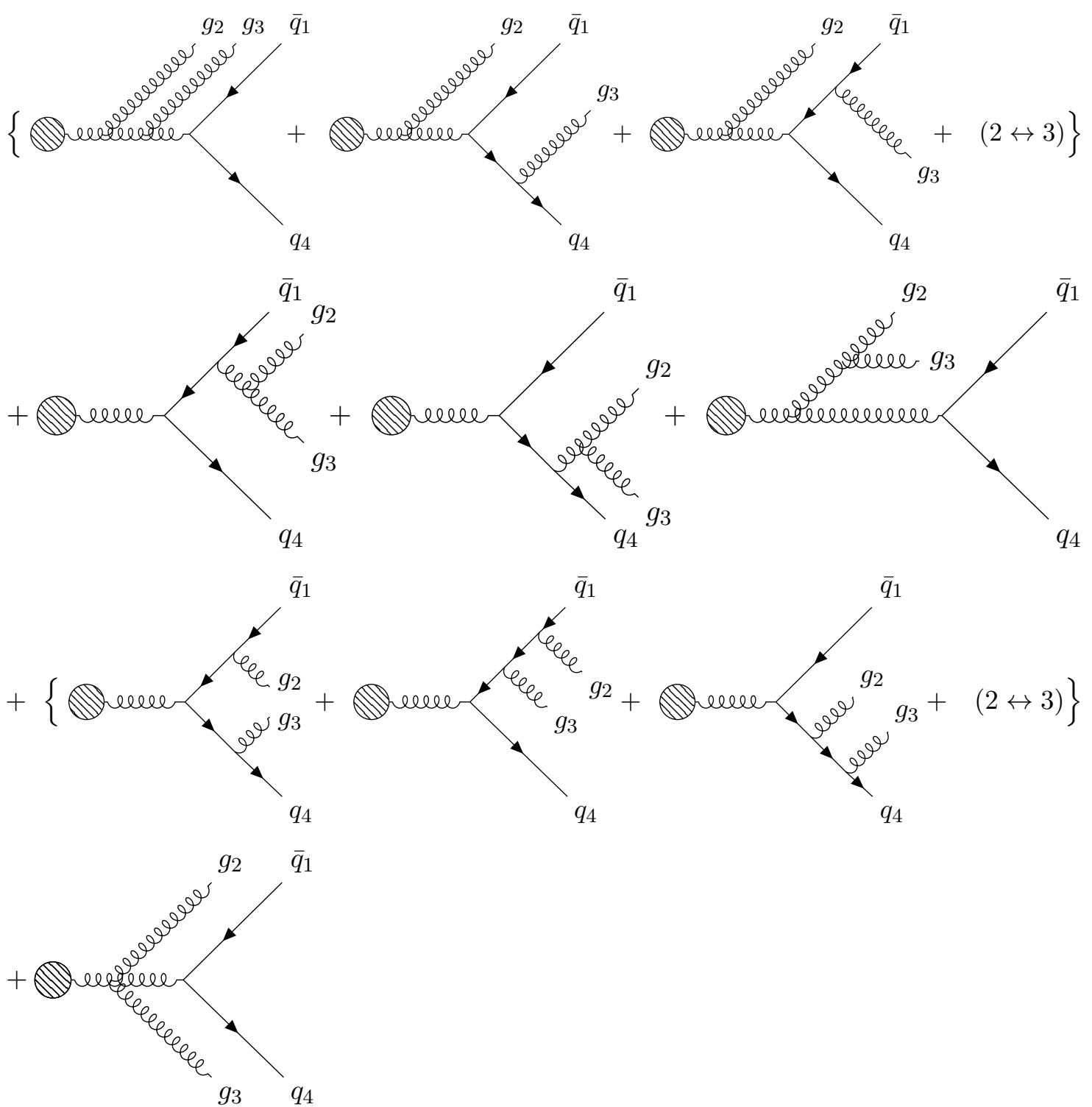

Figure 2. The diagrams contributing to the collinear decay $g \rightarrow \bar{q}_{1} g_{2} g_{3} q_{4}$.

The above permutations do not include orderings of the external gluons which leave the first diagram in figure 3 invariant.

\section{Nested collinear limits}

In this section we analyse the collinear limit of the splitting amplitudes themselves, i.e., we study their behaviour in the limit where a subset of collinear partons is more collinear than the others.

To be concrete, let us consider a collection of $m$ partons with flavour indices $\left\{f_{1}, \ldots, f_{m^{\prime}}, \ldots, f_{m}\right\}$ and momenta $\left\{p_{1}, \ldots, p_{m^{\prime}}, \ldots, p_{m}\right\}$, with $m^{\prime}<m$. We always think 

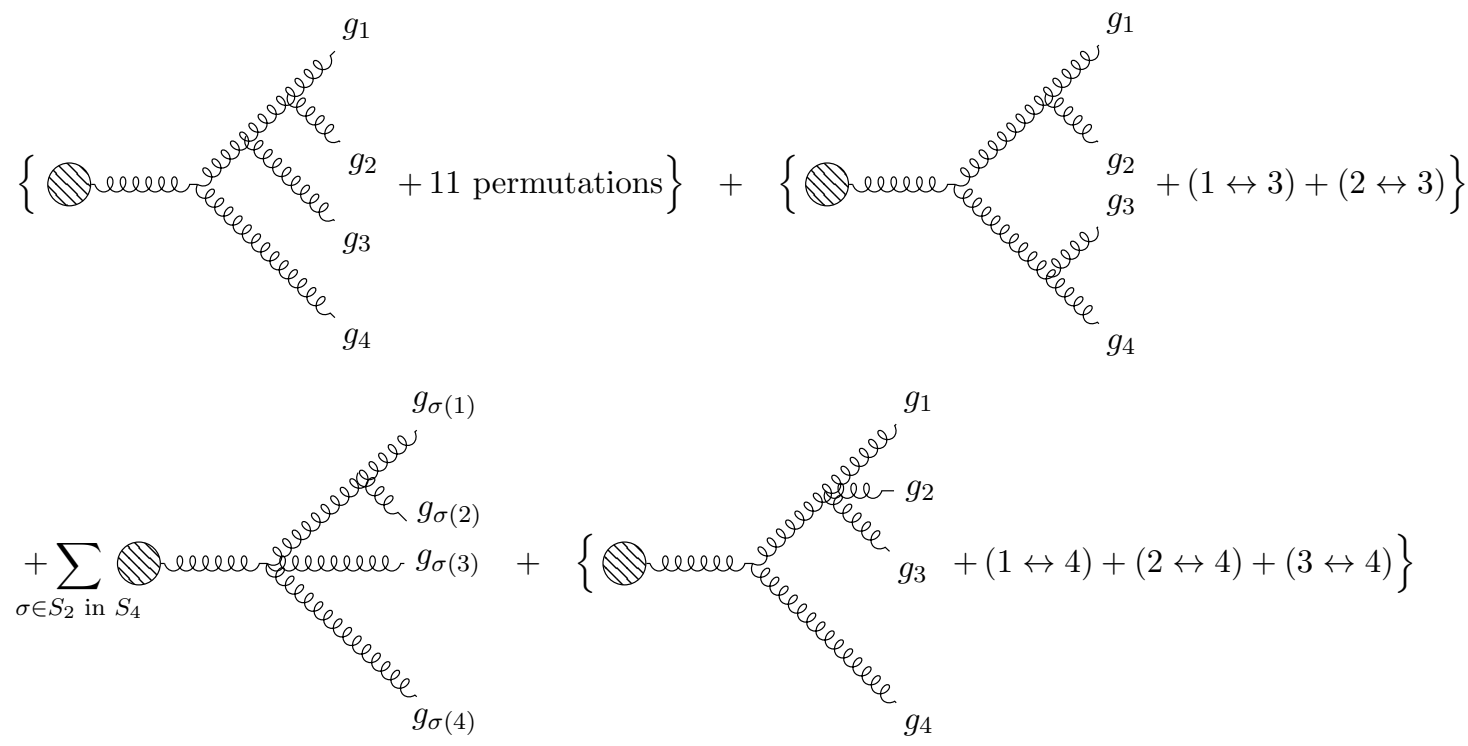

Figure 3. The diagrams contributing to the splitting process $g \rightarrow g_{1} g_{2} g_{3} g_{4}$. In the third diagram we sum over the 6 possible pairings of the partons in the three-gluon vertex.

of these partons as being part of an on-shell $n$-point amplitude $\mathcal{M}_{f_{1} \ldots f_{n}}$ involving $(n-m)$ additional coloured partons. Our goal is to study the behaviour of the amplitude in the limit where $\left\{p_{1}, \ldots, p_{m^{\prime}}\right\}$ become collinear to some lightlike direction $\widetilde{P}^{\prime}$, and $\left\{\widetilde{P}^{\prime}, p_{m^{\prime}+1}, \ldots, p_{m}\right\}$ are collinear to another lightlike direction $\widetilde{P}$. Depending on the order in which the different collinear limits are taken, there are two different scenarios of how such a kinematic configuration can be reached, referred to as iterated and strongly-ordered collinear limits in ref. [73]. The (splitting) amplitudes factorise in the same way in each of the limits, and the factorisation involves the same universal quantities in both cases [73]. We therefore focus here only on the strongly-ordered limit from now on.

We start by giving a precise definition of the strongly-ordered collinear limit. We perform separate light-cone decompositions in each of the $m$ - and $m^{\prime}$-parton sets. For the $m$-parton set, we will use the notations and conventions of eq. (2.1). For the $m^{\prime}$-parton subset we write

$$
p_{i}^{\mu}=y_{i} \widetilde{P}^{\prime \mu}+\kappa_{\perp i}^{\mu}-\frac{\kappa_{\perp i}^{2}}{2 y_{i}} \frac{n^{\prime \mu}}{n^{\prime} \cdot \widetilde{P}^{\prime}}, \quad i=1, \ldots, m^{\prime}
$$

with $n^{\prime 2}=\widetilde{P}^{\prime 2}=\widetilde{P}^{\prime} \cdot \kappa_{\perp i}=n^{\prime} \cdot \kappa_{\perp i}=0$. The momenta $\widetilde{P}$ and $\widetilde{P}^{\prime}$ indicate the directions to which the partons in each set become collinear. We stress that at this point the lightcone directions $\widetilde{P}^{\prime}$ and $n^{\prime}$ in eq. (4.1) are not related to the quantities $\widetilde{P}$ and $n$ in eq. (2.1). However, without loss of generality, we may choose $n^{\prime}=n$, and we work in the axial gauge where the reference vectors of all external and internal gluons are $n$. For more details about the parametrisation of the strongly-ordered collinear limit, we refer to ref. [73]. With this setup, the strongly-ordered collinear limit is defined in analogy with the ordinary collinear limit in section 2: the vectors $k_{\perp i}^{\mu}$ and $\kappa_{\perp i}^{\mu}$ parametrise the transverse distance to the planes 
spanned by $(\widetilde{P}, n)$ and $\left(\widetilde{P}^{\prime}, n\right)$, respectively. The strongly-ordered collinear limit where the $m^{\prime}$-parton subset is more collinear than the $m$-parton set is defined as the limit where both $k_{\perp i}^{\mu}$ and $\kappa_{\perp i}^{\mu}$ approach zero, but the $\kappa_{\perp i}^{\mu}$ tend to zero faster than the $k_{\perp i}^{\mu}$. We can implement the operation of taking this limit by a uniform rescaling of the transverse momenta in each collinear set by a different parameter,

$$
k_{\perp i} \rightarrow \lambda k_{\perp i}, \quad \kappa_{\perp i} \rightarrow \lambda^{\prime} \kappa_{\perp i}
$$

and keeping the dominant singular terms of order $1 / \lambda^{\prime 2\left(m^{\prime}-1\right)} \lambda^{2\left(m-m^{\prime}\right)}$ in the limit $\lambda, \lambda^{\prime} \rightarrow 0$ with $\lambda \gg \lambda^{\prime}$.

The leading behaviour of an amplitude in the strongly-ordered collinear limit is described by a factorisation formula very similar to eq. (2.6) [73],

$$
\begin{gathered}
\mathscr{C}_{\left(1 \ldots m^{\prime}\right) \ldots m} \mathscr{C}_{1 \ldots m^{\prime}}\left|\mathcal{M}_{f_{1} \ldots f_{n}}\left(p_{1}, \ldots, p_{n}\right)\right|^{2}=\left(\frac{2 g_{s}^{2} \mu^{2 \epsilon}}{s_{1 \ldots m^{\prime}}}\right)^{m^{\prime}-1}\left(\frac{2 g_{s}^{2} \mu^{2 \epsilon}}{s_{\left[1 \ldots m^{\prime}\right] \ldots m}}\right)^{m-m^{\prime}} \\
\times \hat{P}_{f_{1} \ldots f_{m^{\prime}}}^{h h^{\prime}} \hat{H}_{f_{\left(1 \ldots m^{\prime}\right)}^{h h^{\prime} ; s s^{\prime}} f_{m^{\prime}+1} \ldots f_{m}} \mathcal{T}_{f f_{m+1}^{s s^{\prime}} \ldots f_{n}}^{s}\left(\widetilde{P}, p_{m^{\prime}+1}, \ldots, p_{n}\right)
\end{gathered}
$$

where

$$
s_{\left[1 \ldots m^{\prime}\right] \ldots m}=\left(\widetilde{P}^{\prime}+p_{m^{\prime}+1}+\ldots+p_{m}\right)^{2} .
$$

The functions $\hat{P}_{f_{1} \ldots f_{m^{\prime}}}^{h h^{\prime}}$ and $\mathcal{T}_{f f_{m+1} \ldots f_{n}}^{s f^{\prime}}$ are the splitting amplitude and the helicity tensor introduced in section 2. The splitting tensor $\hat{H}_{f_{\left(1 \ldots m^{\prime}\right)}^{h h^{\prime} ; s^{\prime}+1} \ldots f_{m}}^{h^{\prime}}$ is new. It is obtained by squaring the amplitude-level splitting amplitude without summing over the helicities of one of the partons in the collinear set (cf. eq. (2.9)),

$$
\begin{aligned}
& \left(\frac{2 g_{s}^{2} \mu^{2 \epsilon}}{s_{\left[1 \ldots m^{\prime}\right] \ldots m}}\right)^{m-m^{\prime}} \hat{H}_{f_{\left(1 \ldots m^{\prime}\right)}^{h h^{\prime} ; s s^{\prime}}}^{f_{m^{\prime}+1} \ldots f_{m}} \\
& =\frac{1}{\mathcal{C}_{f}} \sum_{\substack{\left(s_{m^{\prime}+1}, \ldots, s_{m}\right) \\
\left(c, c_{m^{\prime}+1}, \ldots, c_{m}\right)}} \mathbf{S p}_{f_{\left(1 \ldots m^{\prime}\right)} f_{m^{\prime}+1} \ldots f_{m}}^{c, c_{m^{\prime}+1} \ldots c_{m} ; s, h, s_{m^{\prime}+1} \ldots s_{m}}\left[\mathbf{S p}_{f_{\left(1 \ldots m^{\prime}\right)} f_{m^{\prime}+1} \ldots f_{m}}^{c, c_{m^{\prime}+1} \ldots c_{m} ; s^{\prime}, h^{\prime}, s_{m^{\prime}+1} \ldots s_{m}}\right]^{*},
\end{aligned}
$$

where $\mathcal{C}_{f}$ is defined after eq. (2.9). Just like in section 2 we suppress the dependence of all splitting amplitudes and tensors on their arguments. The factorisation of the squared amplitude in the strongly-ordered limit can be cast in the form of a factorisation of the splitting amplitude itself,

$$
\mathscr{C}_{1 \ldots m^{\prime}} \hat{P}_{f_{1} \ldots f_{m}}^{s s^{\prime}}=\left(\frac{s_{\left[1 \ldots m^{\prime}\right] \ldots m}}{s_{1 \ldots m^{\prime}}}\right)^{m^{\prime}-1} \hat{P}_{f_{1} \ldots f_{m^{\prime}}}^{h h^{\prime}} \hat{H}_{f_{\left(1 \ldots m^{\prime}\right)} f_{m^{\prime}+1} \ldots f_{m}}^{h h^{\prime} ; s^{\prime}} .
$$

By comparing eqs. (2.6) and (4.3), it is easy to see that upon summing over the helicities $\left(h, h^{\prime}\right)$ the splitting tensor reduces to an ordinary splitting amplitude,

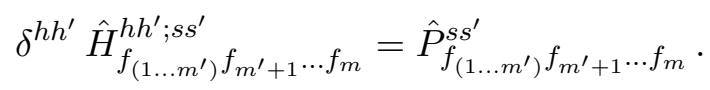

In the following we refer to the partons with spin indices $\left(s, s^{\prime}\right)$ and $\left(h, h^{\prime}\right)$ as the parent and sub-parent, respectively. Depending on the flavour of the parent and the sub-parent, 
the structure of the splitting tensor can be further simplified. The case where the parent is a quark was considered in ref. [73]. Here we only consider the case where the parent is a gluon. Using a similar argument as for the splitting amplitude in section 3, we can trade helicity indices $\left(s, s^{\prime}\right)$ for Lorentz indices $(\mu, \nu)$ and write eq. (4.3) in the equivalent form,

$$
\begin{gathered}
\mathscr{C}_{\left(1 \ldots m^{\prime}\right) \ldots m} \mathscr{C}_{1 \ldots m^{\prime}}\left|\mathcal{M}_{f_{1} \ldots f_{n}}\left(p_{1}, \ldots, p_{n}\right)\right|^{2}=\left(\frac{2 g_{s}^{2} \mu^{2 \epsilon}}{s_{1 \ldots m^{\prime}}}\right)^{m^{\prime}-1}\left(\frac{2 g_{s}^{2} \mu^{2 \epsilon}}{s_{\left[1 \ldots m^{\prime}\right] \ldots m}}\right)^{m-m^{\prime}} \\
\times \hat{P}_{f_{1} \ldots f_{m^{\prime}}}^{h h^{\prime}} \hat{H}_{f_{\left(1 \ldots m^{\prime}\right)}^{h h^{\prime} ; \mu \nu} f_{m^{\prime}+1} \ldots f_{m}} \mathcal{T}_{f f_{m+1} \ldots f_{n}, \mu \nu}\left(\widetilde{P}, p_{m^{\prime}+1}, \ldots, p_{n}\right)
\end{gathered}
$$

If the sub-parent is a quark, helicity must be conserved, and the splitting tensor is diagonal in the spin indices $\left(h, h^{\prime}\right)$ of the sub-parent. Equation (4.7) then implies

$$
\mathscr{C}_{1 \ldots m^{\prime}} \hat{P}_{f_{1} \ldots f_{m}}^{\mu \nu}=\left(\frac{s_{\left[1 \ldots m^{\prime}\right] \ldots m}}{s_{1 \ldots m^{\prime}}}\right)^{m^{\prime}-1}\left\langle\hat{P}_{f_{1} \ldots f_{m^{\prime}}}\right\rangle \hat{P}_{q f_{m^{\prime}+1} \ldots f_{m}}^{\mu \nu},
$$

where $\left\langle\hat{P}_{f_{1} \ldots f_{m^{\prime}}}\right\rangle$ denotes the unpolarised splitting amplitude,

$$
\left\langle\hat{P}_{f_{1} \ldots f_{m}}\right\rangle \equiv \frac{1}{N_{\text {pol }}} \delta_{h h^{\prime}} \hat{P}_{f_{1} \ldots f_{m}}^{h h^{\prime}},
$$

and $N_{\text {pol }}$ denotes the number of physical polarisation states for the parent parton. We work in conventional dimensional regularisation (CDR), where the quarks and gluons have 2 and $(D-2)$ polarisation states, respectively.

If also the sub-parent is a gluon, we can use a similar argument to that of section 3 to trade in the helicity indices $\left(h, h^{\prime}\right)$ for Lorentz indices $(\alpha, \beta)$ by amputating external polarisation vectors and contracting with polarisation tensors. Since only transverse polarisations are physical, only the transverse part of a Lorentz tensor carries physical information. Thus, we can write eq. (4.8) in the equivalent form,

$$
\begin{aligned}
& \mathscr{C}_{\left(1 \ldots m^{\prime}\right) \ldots m} \mathscr{C}_{1 \ldots m^{\prime}}\left|\mathcal{M}_{f_{1} \ldots f_{n}}\left(p_{1}, \ldots, p_{n}\right)\right|^{2}=\left(\frac{2 g_{s}^{2} \mu^{2 \epsilon}}{s_{1 \ldots m^{\prime}}}\right)^{m^{\prime}-1}\left(\frac{2 g_{s}^{2} \mu^{2 \epsilon}}{s_{\left[1 \ldots m^{\prime}\right] \ldots m}}\right)^{m-m^{\prime}} \\
& \times \hat{P}_{f_{1} \ldots f_{m^{\prime}}, \alpha \beta} \hat{H}_{f_{\left(1 \ldots m^{\prime}\right)}^{\alpha \beta ; \mu \nu} f_{m^{\prime}+1} \ldots f_{m}} \mathcal{T}_{f_{f_{m+1} \ldots f_{n}, \mu \nu}}\left(\widetilde{P}, p_{m^{\prime}+1}, \ldots, p_{n}\right),
\end{aligned}
$$

and the relation in eq. (4.7) becomes

$$
\hat{H}_{f_{\left(1 \ldots m^{\prime}\right)}^{\alpha \beta m^{\prime}+1} \ldots f_{m}}^{\alpha \beta \beta} d_{\alpha \beta}\left(\widetilde{P}^{\prime}, n\right)=\hat{P}_{f_{\left(1 \ldots m^{\prime}\right)} f_{m^{\prime}+1} \ldots f_{m}}^{\mu \nu}+\text { gauge terms . }
$$

In appendix B we show that the most general tensor structure of the splitting tensor is

$$
\begin{aligned}
& \hat{H}_{g f_{m^{\prime}+1} \ldots f_{m}}^{\alpha \beta ; \mu \nu}=d^{\alpha \beta}(\widetilde{P}, n) g^{\mu \nu} A_{g f_{m^{\prime}+1} \ldots f_{m}}^{(g)}+\sum_{i, j=m^{\prime}+1}^{m} \frac{k_{\perp i}^{\mu} k_{\perp j}^{\nu}}{s_{\left[1 \ldots m^{\prime}\right] \ldots m}} d^{\alpha \beta}(\widetilde{P}, n) B_{i j, g f_{m^{\prime}+1} \ldots f_{m}}^{(g)} \\
& \quad+\sum_{i, j=m^{\prime}+1}^{m} \frac{k_{\perp i}^{\alpha} k_{\perp j}^{\beta}}{s_{\left[1 \ldots m^{\prime}\right] \ldots m}} g^{\mu \nu} C_{i j, g f_{m^{\prime}+1} \ldots f_{m}}^{(g)}+\sum_{i, j, k, l=m^{\prime}+1}^{m} \frac{k_{\perp i}^{\mu} k_{\perp j}^{\nu} k_{\perp k}^{\alpha} k_{\perp l}^{\beta}}{s_{\left[1 \ldots m^{\prime}\right] \ldots m}^{2}} D_{i j k l, g f_{m^{\prime}+1} \ldots f_{m}}^{(g)} \\
& \quad+\sum_{i, j=m^{\prime}+1}^{m} \frac{g^{\alpha \mu} k_{\perp i}^{\beta} k_{\perp j}^{\nu}+g^{\beta \nu} k_{\perp i}^{\alpha} k_{\perp j}^{\mu}+g^{\alpha \nu} k_{\perp i}^{\beta} k_{\perp j}^{\mu}+g^{\beta \mu} k_{\perp i}^{\alpha} k_{\perp j}^{\nu}}{s_{\left[1 \ldots m^{\prime}\right] \ldots m}} E_{i j, g f_{m^{\prime}+1} \ldots f_{m}}^{(g)} \\
& \quad+\left(g^{\alpha \nu} g^{\beta \mu}+g^{\alpha \mu} g^{\beta \nu}\right) F_{g f_{m^{\prime}+1} \ldots f_{m}}^{(g)} .
\end{aligned}
$$


At this point we have to make some comments about eq. (4.13). First, let us discuss the symmetry properties of the splitting tensor $\hat{H}_{g f_{m^{\prime}+1}^{\alpha} \ldots f_{m}}^{\alpha \beta \mu}$. From its definition in eq. (4.5) it follows that the splitting tensor must be symmetric under the exchange $(\mu, \alpha) \leftrightarrow(\nu, \beta)$. In eq. (4.3) it is contracted with $\hat{P}^{\alpha \beta}$ and $\mathcal{T}^{\mu \nu}$, which are symmetric tensors at tree level. Hence, only the part of $\hat{H}_{g f_{m^{\prime}+1} \ldots f_{m}}^{\alpha \beta \mu \nu}$ that is individually symmetric under $\mu \leftrightarrow \nu$ and $\alpha \leftrightarrow \beta$ enters the factorisation in eq. (4.3), and we only present here the part of the splitting tensor with this enlarged symmetry. Second, we see that eq. (4.13) involves a mixture of metric tensors $g^{\rho \sigma}$ and polarisation tensors $d^{\rho \sigma}(\widetilde{P}, n)$. The tensor structure given in appendix B involves only polarisation tensors, which would make the splitting tensor explicitly transverse. However, similar to the case of the splitting amplitude discussed in section 3 (cf. eq. (3.7)), some of the gauge-dependent terms drop out in the contraction in eq. (4.11). In particular, we can perform the replacements,

$$
d^{\rho \sigma}(\widetilde{P}, n) \leftrightarrow-g^{\rho \sigma}, \quad(\rho, \sigma) \in\{(\mu, \nu),(\alpha, \mu),(\beta, \nu),(\alpha, \nu),(\beta, \mu)\} .
$$

For $(\rho, \sigma)=(\mu, \nu)$, the equivalence between the polarisation tensor and the metric tensor follows from eq. (3.6), while the other cases follow from relations like

$$
\begin{aligned}
\hat{P}_{f_{1} \ldots f_{m^{\prime}}, \alpha \beta} d^{\alpha \mu}(\widetilde{P}, n) d^{\beta \nu}(\widetilde{P}, n) \mathcal{T}_{f f_{m+1} \ldots f_{n}, \mu \nu} & =\hat{P}_{f_{1} \ldots f_{m^{\prime}}, \alpha \beta} g^{\alpha \mu} g^{\beta \nu} \mathcal{T}_{f f_{m+1} \ldots f_{n}, \mu \nu}, \\
\hat{P}_{f_{1} \ldots f_{m^{\prime}}, \alpha \beta} d^{\alpha \mu}(\widetilde{P}, n) k_{\perp i}^{\beta} k_{\perp j}^{\nu} \mathcal{T}_{f f_{m+1} \ldots f_{n}, \mu \nu} & =-\hat{P}_{f_{1} \ldots f_{m^{\prime}}, \alpha \beta} g^{\alpha \mu} k_{\perp i}^{\beta} k_{\perp j}^{\nu} \mathcal{T}_{f_{f_{m+1} \ldots f_{n}, \mu \nu}}
\end{aligned}
$$

We have checked that our results for the quadruple splitting amplitudes have the correct behaviour in all strongly-ordered collinear limits, i.e., they satisfy eq. (4.6) for $m^{\prime}=2$ and 3 . The strongly-ordered limit of the quadruple splitting amplitudes involves the splitting tensors with two or three collinear particles in the final state. The relevant splitting tensors for two collinear partons can be found in ref. [31],

$$
\begin{aligned}
\hat{H}_{\bar{q} q}^{h h^{\prime} ; \mu \nu} & =\frac{1}{2} \delta^{h h^{\prime}} \hat{P}_{\bar{q} q}^{\mu \nu}, \\
\hat{H}_{g g}^{\alpha \beta ; \mu \nu} & =2 C_{A}\left[\frac{1-z}{2 z}\left(g^{\alpha \mu} g^{\beta \nu}+g^{\alpha \nu} g^{\beta \mu}\right)+\frac{z}{1-z} g^{\mu \nu} \frac{k_{\perp}^{\alpha} k_{\perp}^{\beta}}{k_{\perp}^{2}}-z(1-z) \frac{k_{\perp}^{\mu} k_{\perp}^{\nu}}{k_{\perp}^{2}} d^{\alpha \beta}(\widetilde{P}, n)\right],
\end{aligned}
$$

where we set $k_{\perp}=k_{1 \perp}=-k_{2 \perp}$. Note that, compared to ref. [31], we express the splitting tensor $\hat{H}_{g g}^{\alpha \beta ; \mu \nu}$ in a form that is individually symmetric in $(\mu, \nu)$ and $(\alpha, \beta)$. In addition, the two-parton splitting tensor $\hat{H}_{g g}^{\alpha \beta ; \mu \nu}$ is special in that certain tensor structures do not appear. The coefficients $A_{g g}^{(g)}$ and $D_{3333, g g}^{(g)}$ are subleading in the collinear limit, while $E_{33, g g}^{(g)}$ vanishes in the explicitly symmetric form (4.13). The relevant splitting tensors for three collinear partons are new and are given in ref. [73] and in appendix C.

\section{Conclusions}

In this paper, we have computed the quadruple-collinear splitting amplitudes for a gluon parent in CDR. Combined with our previous results for a quark parent [73], this completes the set of tree-level splitting amplitudes describing all collinear singularities for the emission of up to four collinear partons at $\mathrm{N}^{3} \mathrm{LO}$. Our results are available in computer-readable form 
online [74]. We have also considered the strongly-ordered limit when a subset of the four collinear partons become collinear to each other, and we have derived the corresponding factorisation formulæ. Our results satisfy the expected factorisations in all strongly-ordered limits, which provides a strong check on the correctness of our computations.

Our results are an important building block towards understanding the complete infrared structure of massless QCD amplitudes at $\mathrm{N}^{3} \mathrm{LO}$, which is a cornerstone to construct a substraction method at this order. Indeed, firstly, the purely virtual infrared singularities of massless amplitudes with up to three loops are completely known [80-85]. Secondly, when our results are combined with the results for the one-loop emission of up to three collinear particles $[52-58,60,67,68]^{6}$ and the two-loop splitting amplitudes for two collinear partons [61-63], they provide a complete description of all collinear singularities up to $\mathrm{N}^{3} \mathrm{LO}$. Finally, soft emissions are known for the tree-level emission of up to three soft partons [54, 70, 86, 87], and at one and two loops for the emission of a single soft gluon [56, 58, 59, 64-66]. The soft current describing the emission of a pair of two soft partons at one-loop, however, is still missing. For the future, it would be interesting to compute this current and to complete the description of all infrared singularities of massless QCD amplitudes at $\mathrm{N}^{3} \mathrm{LO}$.

\section{Acknowledgments}

CD and MM acknowledge the hospitality of the ETH Zurich, and MM also acknowledges the hospitality of the TH Department of CERN, at various stages of this work. This work was supported in part by the ERC starting grant 637019 "MathAm" (CD), the FRIA grant of the Fonds National de la Recherche Scientifique (FNRS), Belgium (MM), the European Research Council (ERC) under grant agreement No 694712 (PertQCD) and the Swiss National Science Foundation (SNF) under contract agreement No 179016 (VD, RH, AL).

\section{A The iterated collinear limit}

The strongly-ordered amplitude [73],

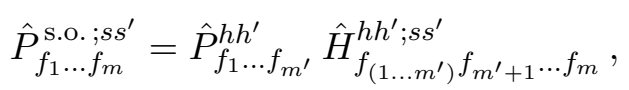

depends on the quantum numbers and light-cone kinematics of both the $m$-parton collinear set and its $m^{\prime}$-parton subset. It is obtained by summing over the helicities $\left(h, h^{\prime}\right)$ of the parent parton of the collinear subset. In case the sub-parent with helicities $\left(h, h^{\prime}\right)$ is a quark, following the factorisation in eq. (4.9), the strongly-ordered splitting amplitude has the same tensor structure as an ordinary splitting amplitude. In case both the parent with helicities $\left(s, s^{\prime}\right)$ and the sub-parent are gluons, the strongly-ordered amplitude has a similar tensor structure as that of a gluon splitting amplitude,

$$
\hat{P}_{f_{1} \ldots f_{m}}^{\text {s.o. } \mu \nu}=g^{\mu \nu} A_{f_{1} \ldots f_{m}}^{\text {s.o. }}+\sum_{i, j=1}^{m^{\prime}} \frac{\kappa_{\perp i}^{\mu} \kappa_{\perp j}^{\nu}}{s_{1 \ldots m^{\prime}}} B_{i j, f_{1} \ldots f_{m}}^{\text {s.o. }}+\sum_{k, l=m^{\prime}+1}^{m} \frac{k_{\perp k}^{\mu} k_{\perp l}^{\nu}}{s_{m^{\prime}+1 \ldots m}} C_{k l, \dot{f}_{1} \ldots f_{m}}^{\text {s.o. }} .
$$

\footnotetext{
${ }^{6}$ But for the one-loop collinear splitting amplitude $q \rightarrow g g q$, which at present is unknown.
} 
It depends on the transverse momenta $\kappa_{\perp i}^{\mu}$ of the collinear subset, $i=1, \ldots, m^{\prime}$ and those of the other $\left(m-m^{\prime}\right)$-collinear partons unaffected by the strongly-ordered limit, $k_{\perp j}^{\mu}$ with $j=m^{\prime}+1, \ldots, m$.

The strongly-ordered splitting amplitude can be obtained by performing the $m^{\prime}$-parton iterated limit on the $m$-parton splitting amplitude,

$$
\hat{P}_{f_{1} \ldots f_{m}}^{\text {s.o. } \mu \nu}=\left(\frac{s_{1 \ldots m^{\prime}}}{s_{\left[1 \ldots m^{\prime}\right] \ldots m}}\right)^{m^{\prime}-1} \mathscr{C}_{1 \ldots m^{\prime}} \hat{P}_{f_{1} \ldots f_{m}}^{\mu \nu} .
$$

The steps involved are essentially the same as for a quark parent, which was considered in ref. [73]. However, as a first step, we need to apply the relation

$$
k_{\perp i}^{\mu}=\zeta_{i} K^{\mu}+\kappa_{\perp i}^{\mu}+\frac{K \cdot \kappa_{\perp i}}{\alpha} \frac{n^{\mu}}{n \cdot \widetilde{P}}, \quad i=1, \ldots, m^{\prime}
$$

where $K^{\mu}=\sum_{j=1}^{m^{\prime}} k_{\perp j}^{\mu}=-\sum_{j=m^{\prime}+1}^{m} k_{\perp j}^{\mu}$ and $\alpha=\sum_{j=1}^{m^{\prime}} z_{j}$. Then, we perform the steps outlined in appendix $\mathrm{C}$ of ref. [73].

Comparing the strongly-ordered limit obtained from eq. (A.3) with that obtained from eq. (A.1), works as a strong check on the splitting amplitudes. This was done to check all possible two- and three-parton sub-limits.

\section{B Tensor structure of gluon parent and sub-parent splitting tensors}

In this appendix, we detail the general tensor structure of the gluon-initiated splitting tensor $\hat{H}_{g f_{m^{\prime}+1} \ldots f_{m}}^{\alpha \beta ; \mu \nu}$ with a gluon sub-parent, defined in section 4. This tensor appears as the collinear limit of the helicity tensor $\mathcal{T}^{\alpha \beta}$, defined in eq. (2.8). Therefore, we begin by considering an $n$-parton tree-level QCD process with its associated helicity tensor $\mathcal{T}_{f_{1} \ldots f_{n}}^{\alpha \beta}\left(p_{1}, \ldots, p_{n}\right)$, where parton 1 carries the Lorentz indices $(\alpha, \beta)$. Using the same kind of argument as for eq. (3.8), our gauge choice allows us to consider only diagrams that have a propagator carrying the momentum $P=\sum_{i=1}^{m} p_{i}$ of the parent gluon. This directly implies that the sub-diagrams splitting the parent parton into $m$ partons will factorise (cf. eq. (3.8)), the only difference being that the spin indices of parton 1 are not summed over,

$$
\begin{aligned}
\mathscr{C}_{1 \ldots m} \mathcal{T}_{f_{1} \ldots f_{n}}^{\alpha \beta} & \left(p_{1}, \ldots, p_{n}\right)= \\
& =\mathscr{C}_{1 \ldots m}\left[\left(\frac{2 \mu^{2 \epsilon} g_{s}^{2}}{s_{1 \ldots m}}\right)^{m-1}\left[\mathcal{M}_{g f_{m+1} \ldots f_{n}}^{(n) \mu}\right]^{*} H_{f_{1} \ldots f_{m}}^{(n) \alpha \beta ; \mu \nu}\left(p_{1}, \ldots, p_{m}\right) \mathcal{M}_{g f_{m+1} \ldots f_{n}}^{(n) \nu}\right] .
\end{aligned}
$$

The squared off-shell current $H_{f_{1} \ldots f_{m}}^{(n) \alpha \beta \mu \nu}$ is the interference of the colour-dressed off-shell currents splitting the parent parton into $m$ collinear partons (cf. eq. (3.9)), with the Lorentz indices of parton 1 and the parent parton open,

$$
\left(\frac{2 \mu^{2 \epsilon} g_{s}^{2}}{s_{1 \ldots m}}\right)^{m-1} H_{f_{1} \ldots f_{m}}^{(n) \alpha \beta ; \mu \nu}=\frac{1}{\mathcal{C}_{f}} \sum_{\substack{\left(s_{2}, \ldots, s_{m}\right) \\\left(c, c_{1}, \ldots, c_{m}\right)}}\left[\mathrm{J}_{f_{1} \ldots f_{m}}^{c, c_{1} \ldots c_{m} ; \nu \beta s_{2} \ldots s_{m}}\right]^{*} \mathrm{~J}_{f_{1} \ldots f_{m}}^{c, c_{1} \ldots c_{m} ; \mu \alpha s_{2} \ldots s_{m}}
$$


where we have suppressed the dependence on the momenta. As usual the superscript $(n)$ indicates the dependence on the reference vector. Given the definition in eq. (B.2), $H_{f_{1} \ldots f_{m}}^{(n) \alpha \beta \mu \nu}$ is symmetric under the simultaneous exchange of the Lorentz indices of parton 1 and the parent parton,

$$
H_{f_{1} \ldots f_{m}}^{(n) \alpha \beta ; \mu \nu}=H_{f_{1} \ldots f_{m}}^{(n) \beta \alpha \mu}
$$

This means that the most general tensor structure for $H_{f_{1} \ldots f_{m}}^{(n) \alpha \beta \mu \nu}$ is given by

$$
\begin{aligned}
H_{g f_{1} \ldots f_{m}}^{(n) \alpha \beta \mu \nu}= & g^{\alpha \beta} g^{\mu \nu} \bar{A}_{g f_{1} \ldots f_{m}}^{(g)}+\sum_{i, j=1}^{m} \frac{p_{i}^{\mu} p_{j}^{\nu}}{s_{1 \ldots m}} g^{\alpha \beta} \bar{B}_{i j, g f_{1} \ldots f_{m}}^{(g)}+\sum_{i, j=1}^{m} \frac{p_{i}^{\alpha} p_{j}^{\beta}}{s_{1 \ldots m}} g^{\mu \nu} \bar{C}_{i j, g f_{1} \ldots f_{m}}^{(g)} \\
& +\sum_{i, j, k, l=1}^{m} \frac{p_{i}^{\mu} p_{j}^{\nu} p_{k}^{\alpha} p_{l}^{\beta}}{s_{1 \ldots m}^{2}} \bar{D}_{i j k l, g f_{1} \ldots f_{m}}^{(g)}+\sum_{i, j=1}^{m} \frac{g^{\alpha \mu} p_{i}^{\beta} p_{j}^{\nu}+g^{\beta \nu} p_{i}^{\alpha} p_{j}^{\mu}}{s_{1 \ldots m}} \bar{E}_{1 i j, g f_{1} \ldots f_{m}}^{(g)} \\
& +\sum_{i, j=1}^{m} \frac{g^{\alpha \nu} p_{i}^{\beta} p_{j}^{\mu}+g^{\beta \mu} p_{i}^{\alpha} p_{j}^{\nu}}{s_{1 \ldots m}} \bar{E}_{2 i j, g f_{m^{\prime}+1} \ldots f_{m}}^{(g)}+g^{\alpha \mu} g^{\beta \nu} \bar{F}_{1 g f_{1} \ldots f_{m}}^{(g)} \\
& +g^{\alpha \nu} g^{\beta \mu} \bar{F}_{2 g f_{1} \ldots f_{m}}^{(g)}+\text { gauge terms }
\end{aligned}
$$

where 'gauge terms' on the right-hand-side denote all terms proportional to the axial gauge vector $n$. By the same arguments as for the tensor structure of the gluon-initiated splitting amplitude, the coefficients $\bar{A}_{g f_{1} \ldots f_{m}}^{(g)}$ through $\bar{F}_{2 g f_{1} \ldots f_{m}}^{(g)}$ in eq. (B.4) are dimensionless and of order zero in the collinear limit. In addition, we have the following symmetries, which follow directly from eq. (B.3),

$$
\begin{aligned}
\bar{B}_{i j, g f_{1} \ldots f_{m}}^{(g)} & =\bar{B}_{j i, g f_{1} \ldots f_{m}}^{(g)}, \\
\bar{C}_{i j, g f_{1} \ldots f_{m}}^{(g)} & =\bar{C}_{j i, g f_{1} \ldots f_{m}}^{(g)}, \\
\bar{D}_{i j k l, g f_{1} \ldots f_{m}}^{(g)} & =\bar{D}_{j i l k, g f_{1} \ldots f_{m}}^{(g)} .
\end{aligned}
$$

Since only the transverse part of a Lorentz tensor holds physical information (cf. sections 3 and 4), we multiply $H_{g f_{1} \ldots f_{m}}^{(n) \alpha \beta \mu \nu}$ by spin-polarisation tensors,

$$
d_{\mu \tilde{\mu}}(P, n) d_{\nu \tilde{\nu}}(P, n) d_{\alpha \tilde{\alpha}}\left(p_{1}, n\right) d_{\beta \tilde{\beta}}\left(p_{1}, n\right) H_{f_{1} \ldots f_{m}}^{(n) \tilde{\beta} \tilde{\mu} \tilde{\nu}}
$$

Next, we take the $m$-parton collinear limit of $H_{f_{1} \ldots f_{m}}^{(n) \alpha \beta \mu \nu}$ to obtain the splitting tensor. Equation (B.1) can then be written as

$$
\mathscr{C}_{1 \ldots m} \mathcal{T}_{f_{1} \ldots f_{n}}^{\alpha \beta}\left(p_{1}, \ldots, p_{n}\right)=\left(\frac{2 \mu^{2 \epsilon} g_{s}^{2}}{s_{1 \ldots m}}\right)^{m-1} \hat{H}_{f_{1} \ldots f_{m}}^{\alpha \beta \mu \nu} \mathcal{T}_{g f_{m+1} \ldots f_{n}, \mu \nu}\left(\widetilde{P}, p_{m+1}, \ldots, p_{n}\right)
$$


By computing explicitly the contractions of the tensors on the right-hand-side of eq. (B.4) with the spin-polarisation tensors, we obtain

$$
\begin{aligned}
d_{\mu \alpha}\left(p_{i}, n\right) g^{\alpha \beta} d_{\nu \beta}\left(p_{i}, n\right) & =-d_{\mu \nu}(\widetilde{P}, n)+\ldots, \\
d^{\mu}{ }_{\nu}\left(p_{j}, n\right) p_{i}^{\nu} & =-k_{\perp i}^{\mu}+\frac{z_{i}}{z_{j}} k_{\perp j}^{\mu}+\ldots, \\
d^{\mu \nu}(P, n) P_{\nu} & =0+\ldots, \\
d^{\mu \nu}(P, n) n_{\nu} & =0 \\
d^{\mu}{ }_{\nu}\left(p_{i}, n\right) \widetilde{P}^{\nu} & =\frac{1}{z_{i}} k_{\perp i}^{\mu}+\ldots, \\
d^{\mu}{ }_{\nu}(\widetilde{P}, n) p_{i}^{\nu} & =-k_{\perp i}^{\mu}+\ldots,
\end{aligned}
$$

where in the ellipses we have suppressed sub-leading terms in the $m$-parton collinear limit. Thus, the transverse part of the splitting tensor in eq. (B.7) contains only combinations of spin-polarisation tensors and transverse momenta.

In addition, we can take into account that in eq. (B.7) the splitting tensor is contracted with the symmetric tensor $\mathcal{T}_{g f_{m+1} \ldots f_{n}}^{\mu \nu}$, which allows us to discard terms in $\hat{H}_{f_{1} \ldots f_{m}}^{\alpha \beta ; \mu \nu}$ that are anti-symmetric under the exchange $\mu \leftrightarrow \nu$. This leads to the following expression for the splitting tensor,

$$
\begin{aligned}
\hat{H}_{g f_{1} \ldots f_{m}}^{\alpha \beta \mu \nu}= & -d^{\alpha \beta} d^{\mu \nu} A_{g f_{1} \ldots f_{m}}^{(g)}+\sum_{i, j=1}^{m} \frac{k_{\perp i}^{\mu} k_{\perp j}^{\nu}}{s_{1 \ldots m}} d^{\alpha \beta} B_{i j, g f_{1} \ldots f_{m}}^{(g)} \\
& -\sum_{i, j=1}^{m} \frac{k_{\perp i}^{\alpha} k_{\perp j}^{\beta}}{s_{1 \ldots m}} d^{\mu \nu} C_{i j, g f_{1} \ldots f_{m}}^{(g)}+\sum_{i, j, k, l=1}^{m} \frac{k_{\perp i}^{\mu} k_{\perp j}^{\nu} k_{\perp k}^{\alpha} k_{\perp l}^{\beta}}{s_{1 \ldots m}^{2}} D_{i j k l, g f_{1} \ldots f_{m}}^{(g)} \\
& -\sum_{i, j=1}^{m} \frac{d^{\alpha \mu} k_{\perp i}^{\beta} k_{\perp j}^{\nu}+d^{\beta \nu} k_{\perp i}^{\alpha} k_{\perp j}^{\mu}+d^{\alpha \nu} k_{\perp i}^{\beta} k_{\perp j}^{\mu}+d^{\beta \mu} k_{\perp i}^{\alpha} k_{\perp j}^{\nu}}{s_{1 \ldots m}} E_{i j, g f_{1} \ldots f_{m}}^{(g)} \\
& +\left(d^{\alpha \mu} d^{\beta \nu}+d^{\alpha \nu} d^{\beta \mu}\right) F_{g f_{1} \ldots f_{m}}^{(g)},
\end{aligned}
$$

where we have suppressed the dependence of the spin-polarisation tensor on the gauge vectors $\widetilde{P}$ and $n$. The scalar coefficients appearing in eq. (B.9) are linear combinations of the coefficients in eq. (B.4). To keep the symmetry of this expression, the coefficients now satisfy

$$
\begin{aligned}
B_{i j, g f_{1} \ldots f_{m}}^{(g)} & =B_{j i, g f_{1} \ldots f_{m}}^{(g)}, \\
C_{i j, g f_{1} \ldots f_{m}}^{(g)} & =C_{j i, g f_{1} \ldots f_{m}}^{(g)}, \\
D_{i j k l, g f_{1} \ldots f_{m}}^{(g)}=D_{j i k l, g f_{1} \ldots f_{m}}^{(g)} & =D_{i j l k, g f_{1} \ldots f_{m}}^{(g)}=D_{j i l k, g f_{1} \ldots f_{m}}^{(g)} .
\end{aligned}
$$

We can now immediately obtain eq. (4.13) from eq. (B.9) by replacing some of the polarisation tensors by metric tensors following the discussion in section 4 .

\section{The three-parton splitting tensors}

There are three gluon-initiated splitting tensors with three partons in the collinear set,

$$
g \rightarrow \bar{q} q g, \quad g \rightarrow g \bar{q} q, \quad g \rightarrow g g g .
$$


The first is obtained by not summing over the helicities $\left(h, h^{\prime}\right)$ of the quark (or equivalently, by charge-conjugation, the anti-quark) in the collinear set. It is proportional to the threeparton splitting amplitude $\hat{P}_{\bar{q} q g}^{\mu \nu}$ given in ref. [54],

$$
\hat{H}_{\bar{q} q g}^{h h^{\prime} ; \mu \nu}=\frac{1}{2} \delta^{h h^{\prime}} \hat{P}_{\bar{q} q g}^{\mu \nu} .
$$

The other two have the tensor structure of eq. (4.13). The three-gluon splitting tensor $\hat{H}_{g g g}^{\alpha \beta, \mu \nu}$ is too lengthy to be presented on paper, but we make it available in computerreadable form [74]. In the remainder of this section, we provide explicit results for the splitting tensor $\hat{H}_{g \bar{q} q}^{\alpha \beta} ; \mu \nu$. We can write it in terms of an 'abelian' and 'non-abelian' part,

$$
\hat{H}_{g \bar{q} q}^{\alpha \beta ; \mu \nu}=\frac{1}{2} C_{F} \hat{H}_{g \bar{q} q}^{\alpha \beta, \mu \nu(\mathrm{ab})}+\frac{1}{2} C_{A} \hat{H}_{g \bar{q} q}^{\alpha \beta, \mu \nu(\mathrm{nab})} .
$$

We add a subscript (12) to denote the on-shell momentum of the gluon sub-parent. Furthermore, we define the shorthand,

$$
z_{1 \ldots j}=z_{1}+\ldots+z_{j}, \quad \bar{z}_{i}=1-z_{i}, \quad k_{\perp 1 \ldots j}=k_{\perp 1}+\ldots+k_{\perp j} .
$$

In what follows, we have eliminated $z_{12}$ and $k_{\perp 12}$ using the constraints. The sub-energies $s_{[i j] k}$ are defined in eq. (4.4). The coefficients in eq. (4.13) belonging to the 'abelian' piece of $\hat{H}_{g_{(12)}}^{\alpha \beta \bar{q}_{3} q_{4}}$ are given by

$$
\begin{aligned}
& A_{g_{(12)}(g) \bar{q}_{3} q_{4}}^{(\mathrm{ab})}=-\frac{\left(s_{[12] 3}+s_{[12] 4}\right)^{2}}{2 s_{[12] 3} s_{[12] 4}}, \\
& B_{33, g_{(12)} \bar{q}_{3} q_{4}}^{(g)(\mathrm{ab})} \equiv B_{34, g_{(12)} \bar{q}_{3} q_{4}}^{(g)(\mathrm{ab})} \equiv B_{43, g_{(12)} \bar{q}_{3} q_{4}}^{(g)(\mathrm{ab})} \equiv B_{44, g_{(12)} \bar{q}_{3} q_{4}}^{(g)(\mathrm{ab})}=-\frac{2 s_{[12] 34}}{s_{[12] 3} s_{[12] 4}}, \\
& C_{33, g_{(12)} \bar{q}_{3} q_{4}}^{(g)(\mathrm{ab})}=-\frac{2 s_{[12] 34}\left(z_{4} s_{[12] 3}-\bar{z}_{4} s_{[12] 4}\right)^{2}}{s_{[12] 3}^{2} s_{[12] 4}^{2}\left(1-z_{34}\right)^{2}}, \\
& C_{44, g_{(12)} \bar{q}_{3} q_{4}}^{(g)(\mathrm{a})}=-\frac{2 s_{[12] 34}\left(z_{3} s_{[12] 4}-\bar{z}_{3} s_{[12] 3}\right)^{2}}{s_{[12] 3}^{2} s_{[12] 4}^{2}\left(1-z_{34}\right)^{2}}, \\
& C_{34, g_{(12)} \bar{q}_{3} q_{4}}^{(g)(\mathrm{ab})} \equiv C_{43, g_{(12)} \bar{q}_{3} q_{4}}^{(g)(\mathrm{ab})}=\frac{2 s_{[12] 34}\left(z_{3} s_{[12] 4}-\bar{z}_{3} s_{[12] 3}\right)\left(z_{4} s_{[12] 3}-\bar{z}_{4} s_{[12] 4}\right)}{s_{[12] 3}^{2} s_{[12] 4}^{2}\left(1-z_{34}\right)^{2}}, \\
& D_{3333, g_{(12)} \bar{q}_{3} q_{4}}^{(g)(\mathrm{ab})}=-\frac{8 z_{4}^{2}}{s_{[12] 4}^{2}\left(1-z_{34}\right)^{2}}, \\
& D_{4444, g_{(12)} \bar{q}_{3} q_{4}}^{(g)(\mathrm{ab})}=-\frac{8 z_{3}{ }^{2}}{s_{[12] 3}^{2}\left(1-z_{34}\right)^{2}}, \\
& D_{3334, g_{(12)}(\mathrm{g}) \bar{q}_{3} q_{4}}^{(g)(\mathrm{a})} \equiv D_{3343, g_{(12)} \bar{q}_{3} q_{4}}^{(g)(\mathrm{ab})}=-\frac{8 z_{4} \bar{z}_{3}}{s_{[12] 4}^{2}\left(1-z_{34}\right)^{2}}, \\
& D_{3433, g_{(12)} \bar{q}_{3} q_{4}}^{(g)(\mathrm{ab})} \equiv D_{4333, g_{(12)} \bar{q}_{3} q_{4}}^{(g)(\mathrm{ab})}=-\frac{8 z_{4} \bar{z}_{4}}{s_{[12] 3} s_{[12] 4}\left(1-z_{34}\right)^{2}}, \\
& D_{4434, g_{(12)} \bar{q}_{3} q_{4}}^{(g)(\mathrm{ab})} \equiv D_{4443, g_{(12)} \bar{q}_{3} q_{4}}^{(g)(\mathrm{ab})}=-\frac{8 z_{3} \bar{z}_{4}}{s_{[12] 3}^{2}\left(1-z_{34}\right)^{2}},
\end{aligned}
$$




$$
\begin{aligned}
D_{3444, g_{(12)} \bar{q}_{3} q_{4}}^{(g)(\mathrm{ab}} & \equiv D_{4344, g_{(12)} \bar{q}_{3} q_{4}}^{(g)(\mathrm{ab})}=-\frac{8 z_{3} \bar{z}_{3}}{s_{[12] 3} s_{[12] 4}\left(1-z_{34}\right)^{2}} \\
D_{3434, g_{(12)} \bar{q}_{3} q_{4}}^{(g)(\mathrm{ab})} & \equiv D_{4343, g_{(12)} \bar{q}_{3} q_{4}}^{(g)(\mathrm{ab})} \equiv D_{4334, g_{(12)} \bar{q}_{3} q_{4}}^{(g)(\mathrm{ab})} \equiv D_{3443, g_{(12)} \bar{q}_{3} q_{4}}^{(g)(\mathrm{ab})} \\
& =4 \frac{z_{3} \bar{z}_{4}+z_{4} \bar{z}_{3}-1}{s_{[12] 3} s_{[12] 4}\left(1-z_{34}\right)^{2}}, \\
D_{4433, g_{(12)} \bar{q}_{3} q_{4}}^{(g)(\mathrm{ab})} & =-\frac{8 \bar{z}_{4}^{2}}{s_{[12] 3}^{2}\left(1-z_{34}\right)^{2}}, \\
D_{3344, g_{(12)} \bar{q}_{3} q_{4}}^{(g)(\mathrm{ab})} & =-\frac{8 \bar{z}_{3}^{2}}{s_{[12] 4}^{2}\left(1-z_{34}\right)^{2}}, \\
E_{33, g_{(12)} \bar{q}_{3} q_{4}}^{(g)(\mathrm{ab})} & =-\frac{2 z_{4}}{s_{[12] 4}\left(1-z_{34}\right)}, \\
E_{44, g_{(12)} \bar{q}_{3} q_{4}}^{(g)(\mathrm{ab})} & =-\frac{2 z_{3}}{s_{[12] 3}\left(1-z_{34}\right)}, \\
E_{34, g_{(12)} \bar{q}_{3} q_{4}}^{(g)(\mathrm{ab})} & =-\frac{2 \bar{z}_{4}}{s_{[12] 3}\left(1-z_{34}\right)} \\
E_{43, g_{(12)} \bar{q}_{3} q_{4}}^{(g)(\mathrm{ab})} & =-\frac{2 \bar{z}_{3}}{s_{[12] 4}\left(1-z_{34}\right)} \\
F_{g_{(12)} \bar{q}_{3} q_{4}}^{(g)(\mathrm{ab})} & =-1, \quad
\end{aligned}
$$

while the 'non-abelian' coefficients read

$$
\begin{aligned}
& A_{g_{(12)}\left(\bar{q}_{3} q_{4}\right.}^{(g)(\mathrm{nab})}=\frac{1}{2}, \\
& B_{33, g_{(12)} \bar{q}_{3} q_{4}}^{(g)(\mathrm{nab})}=\frac{s_{[12] 34}\left(s_{34}+s_{[12] 4}\right)}{s_{34} s_{[12] 3} s_{[12] 4}}, \\
& B_{34, g_{(12)} \bar{q}_{3} q_{4}}^{(g)(\mathrm{nab}} \equiv B_{43, g_{(12)} \bar{q}_{3} q_{4}}^{(g)(\mathrm{nab})}=\frac{s_{[12] 34}\left(s_{[12] 34}+s_{34}\right)}{2 s_{34} s_{[12] 3} s_{[12] 4}} \\
& B_{44, g_{(12)} \bar{q}_{3} q_{4}}^{(g)(\mathrm{nab})}=\frac{s_{[12] 34}\left(s_{34}+s_{[12] 3}\right)}{s_{34} s_{[12] 3} s_{[12] 4}}, \\
& C_{33, g_{(12)} \bar{q}_{3} q_{4}}^{(g)(\mathrm{nab}}=-\frac{s_{[12] 34}\left(\bar{z}_{4}\left(2 z_{4} s_{34}+s_{[12] 4}\right)+z_{4} s_{[12] 3}\right)}{s_{34} s_{[12] 3} s_{[12] 4}\left(1-z_{34}\right)^{2}}, \\
& C_{44, g_{(12)} \bar{q}_{3} q_{4}}^{(g)(\mathrm{nab})}=-\frac{s_{[12] 34}\left(\bar{z}_{3}\left(2 z_{3} s_{34}+s_{[12] 3}\right)+z_{3} s_{[12] 4}\right)}{s_{34} s_{[12] 3} s_{[12] 4}\left(1-z_{34}\right)^{2}}, \\
& C_{34, g_{(12)} \bar{q}_{3} q_{4}}^{(g)(\mathrm{nab}} \equiv C_{43, g_{(12)} \bar{q}_{3} q_{4}}^{(g)(\mathrm{nab})}=-\frac{s_{[12] 34}}{\left(1-z_{34}\right)^{2}}\left(\frac{\bar{z}_{4} \bar{z}_{3}+z_{4} z_{3}}{s_{[12] 3} s_{[12] 4}}+\frac{\bar{z}_{3}+z_{4}}{2 s_{34} s_{[12] 4}}+\frac{\bar{z}_{4}+z_{3}}{2 s_{34} s_{[12] 3}}\right), \\
& D_{3333, g_{(12)} \bar{q}_{3} q_{4}}^{(g)(\mathrm{nab}}=-\frac{8 z_{4}^{2}\left(s_{34}+s_{[12] 4}\right)}{s_{34}{ }^{2} s_{[12] 4}\left(1-z_{34}\right)^{2}}, \\
& D_{4444, g_{(12)} \bar{q}_{3} q_{4}}^{(g)(\mathrm{nab}}=-\frac{8 z_{3}^{2}\left(s_{34}+s_{[12] 3}\right)}{s_{34}{ }^{2} s_{[12] 3}\left(1-z_{34}\right)^{2}}, \\
& D_{3334, g_{(12)} \bar{q}_{3} q_{4}}^{(g)(\mathrm{nab})} \equiv D_{3343, g_{(12)} \bar{q}_{3} q_{4}}^{(g)(\mathrm{nab})}=-\frac{8 z_{4} \bar{z}_{3}\left(s_{34}+s_{[12] 4}\right)}{s_{34}{ }^{2} s_{[12] 4}\left(1-z_{34}\right)^{2}},
\end{aligned}
$$




$$
\begin{aligned}
& D_{3433, g_{(12)} \bar{q}_{3} q_{4}}^{(g)(\mathrm{nab}} \equiv D_{4333, g_{(12)} \bar{q}_{3} q_{4}}^{(g)(\mathrm{nab})}=\frac{4 z_{4} \bar{z}_{4}}{s_{[12] 4}\left(1-z_{34}\right)^{2}}\left(\frac{s_{34}+2 s_{[12] 4}}{s_{34}^{2}}+\frac{s_{34}+s_{[12] 4}}{s_{34} s_{[12] 3}}\right), \\
& D_{4434, g_{(12)} \bar{q}_{3} q_{4}}^{(g)(\mathrm{nab})} \equiv D_{4443, g_{(12)} \bar{q}_{3} q_{4}}^{(g)(\mathrm{nab})}=-\frac{8 z_{3} \bar{z}_{4}\left(s_{34}+s_{[12] 3}\right)}{s_{34}{ }^{2} s_{[12] 3}\left(1-z_{34}\right)^{2}}, \\
& D_{3444, g_{(12)} \bar{q}_{3} q_{4}}^{(g)(\mathrm{nab}} \equiv D_{4344, g_{(12)} \bar{q}_{3} q_{4}}^{(g)(\mathrm{nab})}=\frac{4 z_{3} \bar{z}_{3}}{s_{[12] 4}\left(1-z_{34}\right)^{2}}\left(\frac{s_{34}+2 s_{[12] 4}}{s_{34}^{2}}+\frac{s_{34}+s_{[12] 4}}{s_{34} s_{[12] 3}}\right) \text {, } \\
& D_{3434, g_{(12)} \bar{q}_{3} q_{4}}^{(g)(\mathrm{nab})} \equiv D_{4343, g_{(12)} \bar{q}_{3} q_{4}}^{(g)(\mathrm{nab})} \equiv D_{4334, g_{(12)} \bar{q}_{3} q_{4}}^{(g)(\mathrm{na})} \equiv D_{3443, g_{(12)} \bar{q}_{3} q_{4}}^{(g)(\mathrm{nab}} \\
& =2 \frac{\bar{z}_{4} \bar{z}_{3}+z_{3} z_{4}}{\left(1-z_{34}\right)^{2}}\left(\frac{2}{s_{34}^{2}}+\frac{s_{[12] 34}}{s_{[12] 3} s_{[12] 4} s_{34}}\right), \\
& D_{4433, g_{(12)} \bar{q}_{3} q_{4}}^{(g)(\mathrm{nab}}=-\frac{8 \bar{z}_{4}^{2}\left(s_{34}+s_{[12] 3}\right)}{s_{34}^{2} s_{[12] 3}\left(1-z_{34}\right)^{2}}, \\
& D_{3344, g_{(12)} \bar{q}_{3} q_{4}}^{(g)(\mathrm{nab}}=-\frac{8 \bar{z}_{3}^{2}\left(s_{34}+s_{[12] 4}\right)}{s_{34}{ }^{2} s_{[12] 4}\left(1-z_{34}\right)^{2}}, \\
& E_{33, g_{(12)} \bar{q}_{3} q_{4}}^{(g)(\mathrm{nab})}=\frac{2 z_{4}^{2}\left(s_{[12] 3}+s_{34}\right)-4 z_{3} z_{4} s_{[12] 4}}{\left(1-z_{34}\right) z_{34} s_{34}}\left(\frac{1}{s_{[12] 4}}+\frac{1}{s_{34}}\right)+\frac{2 z_{4}^{2} s_{[12] 3}}{\left(1-z_{34}\right) z_{34} s_{34}^{2}}, \\
& E_{44, g_{(12)} \bar{q}_{3} q_{4}}^{(g)(\mathrm{nab})}=\frac{2 z_{3}^{2}\left(s_{[12] 4}+s_{34}\right)-4 z_{3} z_{4} s_{[12] 3}}{\left(1-z_{34}\right) z_{34} s_{34}}\left(\frac{1}{s_{[12] 3}}+\frac{1}{s_{34}}\right)+\frac{2 z_{3}^{2} s_{[12] 4}}{\left(1-z_{34}\right) z_{34} s_{34}^{2}}, \\
& E_{34, g_{(12)} \bar{q}_{3} q_{4}}^{(g)(\mathrm{nab})}=\frac{s_{34}+s_{[12] 4}}{2 s_{34} s_{[12] 3} z_{34}}-\frac{\left(s_{34}+s_{[12] 3}\right)}{2 s_{34} s_{[12] 4}}\left(\frac{4 z_{4} \bar{z}_{3}}{\left(1-z_{34}\right) z_{34}}+\frac{1}{z_{34}}\right) \\
& +\frac{4 \bar{z}_{3}}{s_{34}\left(1-z_{34}\right) z_{34}}\left(z_{3}-\frac{1}{2} z_{4}-\frac{s_{[12] 3} z_{4}-s_{[12] 4} z_{3}}{s_{34}}\right), \\
& E_{43, g_{(12)} \bar{q}_{3} q_{4}}^{(g)(\mathrm{nab})}=\frac{s_{34}+s_{[12] 3}}{2 s_{34} s_{[12] 4} z_{34}}-\frac{\left(s_{34}+s_{[12] 4}\right)}{2 s_{34} s_{[12] 3}}\left(\frac{4 z_{3} \bar{z}_{4}}{\left(1-z_{34}\right) z_{34}}+\frac{1}{z_{34}}\right) \\
& +\frac{4 \bar{z}_{4}}{s_{34}\left(1-z_{34}\right) z_{34}}\left(z_{4}-\frac{1}{2} z_{3}+\frac{s_{[12] 3} z_{4}-s_{[12] 4} z_{3}}{s_{34}}\right), \\
& F_{g_{(12)}(g)\left(\bar{q}_{3} q_{4}\right.}^{(n a b)}=\frac{1}{4}-\frac{\left(z_{3} s_{[12] 4}-z_{4} s_{[12] 3}\right)^{2}}{s_{34}{ }^{2} z_{34}^{2}}+\frac{\left(s_{34}+s_{[12] 4}\right)\left(s_{34}-z_{3} s_{[12] 4}\right)}{2 s_{34} s_{[12] 3} z_{34}}-\frac{s_{34}+s_{[12] 3}}{2 s_{[12] 4}} \\
& +\frac{s_{[12] 4}\left(z_{34}\left(4-3 z_{4}\right)-8 z_{3}^{2}\right)}{2 s_{34} z_{34}^{2}}+\frac{3\left(1-z_{34}\right)}{2 z_{34}}+\frac{z_{3} z_{4}}{z_{34}^{2}}+(3 \leftrightarrow 4) .
\end{aligned}
$$

Open Access. This article is distributed under the terms of the Creative Commons Attribution License (CC-BY 4.0), which permits any use, distribution and reproduction in any medium, provided the original author(s) and source are credited.

\section{References}

[1] ATLAS collaboration, Observation of a new particle in the search for the Standard Model Higgs boson with the ATLAS detector at the LHC, Phys. Lett. B 716 (2012) 1 [arXiv: 1207.7214] [inSPIRE].

[2] CMS collaboration, Observation of a new boson at a mass of $125 \mathrm{GeV}$ with the CMS experiment at the LHC, Phys. Lett. B $\mathbf{7 1 6}$ (2012) 30 [arXiv:1207.7235] [InSPIRE]. 
[3] D. Graudenz, M. Spira and P.M. Zerwas, QCD corrections to Higgs boson production at proton proton colliders, Phys. Rev. Lett. 70 (1993) 1372 [INSPIRE].

[4] M. Spira, A. Djouadi, D. Graudenz and P.M. Zerwas, Higgs boson production at the LHC, Nucl. Phys. B 453 (1995) 17 [hep-ph/9504378] [INSPIRE].

[5] C. Anastasiou, C. Duhr, F. Dulat, F. Herzog and B. Mistlberger, Higgs boson gluon-fusion production in QCD at three loops, Phys. Rev. Lett. 114 (2015) 212001 [arXiv:1503.06056] [INSPIRE].

[6] C. Anastasiou et al., High precision determination of the gluon fusion Higgs boson cross-section at the LHC, JHEP 05 (2016) 058 [arXiv: 1602.00695] [INSPIRE].

[7] B. Mistlberger, Higgs boson production at hadron colliders at $N^{3} L O$ in QCD, JHEP 05 (2018) 028 [arXiv: 1802.00833] [INSPIRE].

[8] C. Duhr, F. Dulat and B. Mistlberger, Higgs production in bottom-quark fusion to third order in the strong coupling, Phys. Rev. Lett. 125 (2020) 051804 [arXiv: 1904.09990] [INSPIRE].

[9] C. Duhr, F. Dulat, V. Hirschi and B. Mistlberger, Higgs production in bottom quark fusion: matching the 4- and 5-flavour schemes to third order in the strong coupling, JHEP 08 (2020) 017 [arXiv: 2004.04752] [INSPIRE].

[10] F.A. Dreyer and A. Karlberg, Vector-boson fusion Higgs production at three loops in QCD, Phys. Rev. Lett. 117 (2016) 072001 [arXiv: 1606.00840] [InSPIRE].

[11] F.A. Dreyer and A. Karlberg, Vector-boson fusion Higgs pair production at $N^{3} L O$, Phys. Rev. D 98 (2018) 114016 [arXiv: 1811.07906] [INSPIRE].

[12] L.-B. Chen, H.T. Li, H.-S. Shao and J. Wang, Higgs boson pair production via gluon fusion at $N^{3} L O$ in QCD, Phys. Lett. B 803 (2020) 135292 [arXiv:1909.06808].

[13] L.-B. Chen, H.T. Li, H.-S. Shao and J. Wang, The gluon-fusion production of Higgs boson pair: $N^{3} L O Q C D$ corrections and top-quark mass effects, JHEP 03 (2020) 072 [arXiv: 1912.13001] [INSPIRE].

[14] C. Duhr, F. Dulat and B. Mistlberger, The Drell-Yan cross section to third order in the strong coupling constant, arXiv:2001.07717 [INSPIRE].

[15] L. Cieri, X. Chen, T. Gehrmann, E.W.N. Glover and A. Huss, Higgs boson production at the LHC using the $q_{T}$ subtraction formalism at $N^{3} L O Q C D$, JHEP 02 (2019) 096 [arXiv: 1807.11501] [INSPIRE].

[16] F. Dulat, B. Mistlberger and A. Pelloni, Precision predictions at $N^{3} L O$ for the Higgs boson rapidity distribution at the LHC, Phys. Rev. D 99 (2019) 034004 [arXiv:1810.09462] [INSPIRE].

[17] T. Binoth and G. Heinrich, An automatized algorithm to compute infrared divergent multiloop integrals, Nucl. Phys. B 585 (2000) 741 [hep-ph/0004013] [INSPIRE].

[18] C. Anastasiou and A. Daleo, Numerical evaluation of loop integrals, JHEP 10 (2006) 031 [hep-ph/0511176] [INSPIRE].

[19] S. Catani and M. Grazzini, An NNLO subtraction formalism in hadron collisions and its application to Higgs boson production at the LHC, Phys. Rev. Lett. 98 (2007) 222002 [hep-ph/0703012] [INSPIRE].

[20] R. Boughezal, X. Liu and F. Petriello, $N$-jettiness soft function at next-to-next-to-leading order, Phys. Rev. D 91 (2015) 094035 [arXiv:1504.02540] [INSPIRE]. 
[21] J. Gaunt, M. Stahlhofen, F.J. Tackmann and J.R. Walsh, $N$-jettiness subtractions for NNLO QCD calculations, JHEP 09 (2015) 058 [arXiv: 1505.04794] [INSPIRE].

[22] A. Gehrmann-De Ridder, T. Gehrmann and E.W. Glover, Antenna subtraction at NNLO, JHEP 09 (2005) 056 [hep-ph/0505111] [INSPIRE].

[23] A. Daleo, T. Gehrmann and D. Maître, Antenna subtraction with hadronic initial states, JHEP 04 (2007) 016 [hep-ph/0612257] [INSPIRE].

[24] A. Gehrmann-De Ridder, T. Gehrmann and E.W. Glover, Gluon-gluon antenna functions from Higgs boson decay, Phys. Lett. B 612 (2005) 49 [hep-ph/0502110] [InSPIRE].

[25] A. Gehrmann-De Ridder, T. Gehrmann and E.W. Glover, Quark-gluon antenna functions from neutralino decay, Phys. Lett. B 612 (2005) 36 [hep-ph/0501291] [INSPIRE].

[26] A. Daleo, A. Gehrmann-De Ridder, T. Gehrmann and G. Luisoni, Antenna subtraction at NNLO with hadronic initial states: initial-final configurations, JHEP 01 (2010) 118 [arXiv: 0912.0374] [INSPIRE].

[27] T. Gehrmann and P.F. Monni, Antenna subtraction at NNLO with hadronic initial states: real-virtual initial-initial configurations, JHEP 12 (2011) 049 [arXiv:1107.4037] [INSPIRE].

[28] R. Boughezal, A. Gehrmann-De Ridder and M. Ritzmann, Antenna subtraction at NNLO with hadronic initial states: double real radiation for initial-initial configurations with two quark flavours, JHEP 02 (2011) 098 [arXiv: 1011.6631] [INSPIRE].

[29] A. Gehrmann-De Ridder, T. Gehrmann and M. Ritzmann, Antenna subtraction at NNLO with hadronic initial states: double real initial-initial configurations, JHEP 10 (2012) 047 [arXiv: 1207.5779] [INSPIRE].

[30] J. Currie, E.W.N. Glover and S. Wells, Infrared structure at NNLO using antenna subtraction, JHEP 04 (2013) 066 [arXiv:1301.4693] [INSPIRE].

[31] G. Somogyi, Z. Trócsányi and V. Del Duca, Matching of singly- and doubly-unresolved limits of tree-level QCD squared matrix elements, JHEP 06 (2005) 024 [hep-ph/0502226] [INSPIRE].

[32] G. Somogyi, Z. Trócsányi and V. Del Duca, A subtraction scheme for computing QCD jet cross sections at NNLO: regularization of doubly-real emissions, JHEP 01 (2007) 070 [hep-ph/0609042] [INSPIRE].

[33] G. Somogyi and Z. Trócsányi, A subtraction scheme for computing QCD jet cross sections at NNLO: regularization of real-virtual emission, JHEP 01 (2007) 052 [hep-ph/0609043] [INSPIRE].

[34] G. Somogyi and Z. Trócsányi, A subtraction scheme for computing QCD jet cross sections at NNLO: integrating the subtraction terms. I, JHEP 08 (2008) 042 [arXiv:0807.0509] [INSPIRE].

[35] U. Aglietti, V. Del Duca, C. Duhr, G. Somogyi and Z. Trócsányi, Analytic integration of real-virtual counterterms in NNLO jet cross sections. I, JHEP 09 (2008) 107 [arXiv: 0807.0514] [INSPIRE].

[36] G. Somogyi, Subtraction with hadronic initial states at NLO: an NNLO-compatible scheme, JHEP 05 (2009) 016 [arXiv:0903.1218] [INSPIRE]. 
[37] P. Bolzoni, S.-O. Moch, G. Somogyi and Z. Trócsányi, Analytic integration of real-virtual counterterms in NNLO jet cross sections. II, JHEP 08 (2009) 079 [arXiv:0905.4390] [INSPIRE].

[38] P. Bolzoni, G. Somogyi and Z. Trócsányi, A subtraction scheme for computing QCD jet cross sections at NNLO: integrating the iterated singly-unresolved subtraction terms, JHEP 01 (2011) 059 [arXiv: 1011.1909] [INSPIRE].

[39] V. Del Duca, G. Somogyi and Z. Trócsányi, Integration of collinear-type doubly unresolved counterterms in NNLO jet cross sections, JHEP 06 (2013) 079 [arXiv:1301.3504] [INSPIRE].

[40] G. Somogyi, A subtraction scheme for computing QCD jet cross sections at NNLO: integrating the doubly unresolved subtraction terms, JHEP 04 (2013) 010 [arXiv:1301.3919] [INSPIRE].

[41] M. Czakon, A novel subtraction scheme for double-real radiation at NNLO, Phys. Lett. B 693 (2010) 259 [arXiv:1005.0274] [INSPIRE].

[42] M. Czakon, Double-real radiation in hadronic top quark pair production as a proof of a certain concept, Nucl. Phys. B 849 (2011) 250 [arXiv:1101.0642] [INSPIRE].

[43] M. Czakon and D. Heymes, Four-dimensional formulation of the sector-improved residue subtraction scheme, Nucl. Phys. B 890 (2014) 152 [arXiv:1408.2500] [INSPIRE].

[44] M. Czakon, A. van Hameren, A. Mitov and R. Poncelet, Single-jet inclusive rates with exact color at $O\left(\alpha_{s}^{4}\right)$, JHEP 10 (2019) 262 [arXiv: 1907.12911] [INSPIRE].

[45] F. Caola, K. Melnikov and R. Röntsch, Nested soft-collinear subtractions in NNLO QCD computations, Eur. Phys. J. C $\mathbf{7 7}$ (2017) 248 [arXiv:1702.01352] [INSPIRE].

[46] F. Caola, M. Delto, H. Frellesvig and K. Melnikov, The double-soft integral for an arbitrary angle between hard radiators, Eur. Phys. J. C 78 (2018) 687 [arXiv:1807.05835] [INSPIRE].

[47] M. Delto and K. Melnikov, Integrated triple-collinear counter-terms for the nested soft-collinear subtraction scheme, JHEP 05 (2019) 148 [arXiv:1901.05213] [INSPIRE].

[48] F. Caola, K. Melnikov and R. Röntsch, Analytic results for color-singlet production at NNLO QCD with the nested soft-collinear subtraction scheme, Eur. Phys. J. C $\mathbf{7 9}$ (2019) 386 [arXiv: 1902.02081] [INSPIRE].

[49] F. Caola, K. Melnikov and R. Röntsch, Analytic results for decays of color singlets to gg and $q \bar{q}$ final states at NNLO QCD with the nested soft-collinear subtraction scheme, Eur. Phys. J. C 79 (2019) 1013 [arXiv: 1907.05398] [INSPIRE].

[50] M. Cacciari, F.A. Dreyer, A. Karlberg, G.P. Salam and G. Zanderighi, Fully differential vector-boson-fusion Higgs production at next-to-next-to-leading order, Phys. Rev. Lett. 115 (2015) 082002 [Erratum ibid. 120 (2018) 139901] [arXiv: 1506.02660] [INSPIRE].

[51] J. Currie, T. Gehrmann, E.W.N. Glover, A. Huss, J. Niehues and A. Vogt, $N^{3} L O$ corrections to jet production in deep inelastic scattering using the Projection-to-Born method, JHEP 05 (2018) 209 [arXiv: 1803.09973] [INSPIRE].

[52] Z. Bern, L.J. Dixon, D.C. Dunbar and D.A. Kosower, One loop $N$ point gauge theory amplitudes, unitarity and collinear limits, Nucl. Phys. B 425 (1994) 217 [hep-ph/9403226] [INSPIRE].

[53] J.M. Campbell and E.W. Glover, Double unresolved approximations to multiparton scattering amplitudes, Nucl. Phys. B $\mathbf{5 2 7}$ (1998) 264 [hep-ph/9710255] [INSPIRE]. 
[54] S. Catani and M. Grazzini, Infrared factorization of tree level QCD amplitudes at the next-to-next-to-leading order and beyond, Nucl. Phys. B 570 (2000) 287 [hep-ph/9908523] [INSPIRE].

[55] V. Del Duca, A. Frizzo and F. Maltoni, Factorization of tree QCD amplitudes in the high-energy limit and in the collinear limit, Nucl. Phys. B 568 (2000) 211 [hep-ph/9909464] [INSPIRE].

[56] Z. Bern, V. Del Duca and C.R. Schmidt, The infrared behavior of one loop gluon amplitudes at next-to-next-to-leading order, Phys. Lett. B 445 (1998) 168 [hep-ph/9810409] [INSPIRE].

[57] D.A. Kosower and P. Uwer, One loop splitting amplitudes in gauge theory, Nucl. Phys. B 563 (1999) 477 [hep-ph/9903515] [INSPIRE].

[58] Z. Bern, V. Del Duca, W.B. Kilgore and C.R. Schmidt, The infrared behavior of one loop QCD amplitudes at next-to-next-to leading order, Phys. Rev. D 60 (1999) 116001 [hep-ph/9903516] [INSPIRE].

[59] S. Catani and M. Grazzini, The soft gluon current at one loop order, Nucl. Phys. B 591 (2000) 435 [hep-ph/0007142] [inSPIRE].

[60] D.A. Kosower, Multiple singular emission in gauge theories, Phys. Rev. D 67 (2003) 116003 [hep-ph/0212097] [INSPIRE].

[61] Z. Bern, L.J. Dixon and D.A. Kosower, Two-loop $g \rightarrow$ gg splitting amplitudes in QCD, JHEP 08 (2004) 012 [hep-ph/0404293] [INSPIRE].

[62] S.D. Badger and E.W. Glover, Two loop splitting functions in QCD, JHEP 07 (2004) 040 [hep-ph/0405236] [INSPIRE].

[63] C. Duhr, T. Gehrmann and M. Jaquier, Two-loop splitting amplitudes and the single-real contribution to inclusive Higgs production at $N^{3}$ LO, JHEP 02 (2015) 077 [arXiv: 1411.3587] [INSPIRE].

[64] C. Duhr and T. Gehrmann, The two-loop soft current in dimensional regularization, Phys. Lett. B 727 (2013) 452 [arXiv: 1309.4393] [INSPIRE].

[65] Y. Li and H.X. Zhu, Single soft gluon emission at two loops, JHEP 11 (2013) 080 [arXiv: 1309.4391] [INSPIRE].

[66] L.J. Dixon, E. Herrmann, K. Yan and H.X. Zhu, Soft gluon emission at two loops in full color, JHEP 05 (2020) 135 [arXiv: 1912.09370] [INSPIRE].

[67] S. Catani, D. de Florian and G. Rodrigo, The triple collinear limit of one loop QCD amplitudes, Phys. Lett. B 586 (2004) 323 [hep-ph/0312067] [INSPIRE].

[68] S. Badger, F. Buciuni and T. Peraro, One-loop triple collinear splitting amplitudes in QCD, JHEP 09 (2015) 188 [arXiv: 1507.05070] [INSPIRE].

[69] G.F.R. Sborlini, D. de Florian and G. Rodrigo, Triple collinear splitting functions at NLO for scattering processes with photons, JHEP 10 (2014) 161 [arXiv:1408.4821] [INSPIRE].

[70] S. Catani, D. Colferai and A. Torrini, Triple (and quadruple) soft-gluon radiation in $Q C D$ hard scattering, JHEP 01 (2020) 118 [arXiv:1908.01616] [INSPIRE].

[71] T.G. Birthwright, E.W. Glover, V.V. Khoze and P. Marquard, Multi-gluon collinear limits from MHV diagrams, JHEP 05 (2005) 013 [hep-ph/0503063] [INSPIRE].

[72] C. Duhr, Applications of twistor methods in QCD, master's thesis, Université Catholique de Louvain, Ottignies-Louvain-la-Neuve, Belgium (2006). 
[73] V. Del Duca, C. Duhr, R. Haindl, A. Lazopoulos and M. Michel, Tree-level splitting amplitudes for a quark into four collinear partons, JHEP 02 (2020) 189 [arXiv:1912.06425] [INSPIRE].

[74] "Quadruple collinear splitting amplitudes" supplementary material, https://people.phys.ethz.ch/ pheno/quadruple_collinear.

[75] D. Amati, R. Petronzio and G. Veneziano, Relating hard QCD processes through universality of mass singularities, Nucl. Phys. B 140 (1978) 54 [inSPIRE].

[76] D. Amati, R. Petronzio and G. Veneziano, Relating hard QCD processes through universality of mass singularities. II, Nucl. Phys. B 146 (1978) 29 [INSPIRE].

[77] R. Ellis, H. Georgi, M. Machacek, H. Politzer and G.G. Ross, Factorization and the parton model in QCD, Phys. Lett. B 78 (1978) 281 [INSPIRE].

[78] S. Catani, D. de Florian and G. Rodrigo, Space-like (versus time-like) collinear limits in QCD: is factorization violated?, JHEP 07 (2012) 026 [arXiv:1112.4405] [INSPIRE].

[79] D. de Florian and M. Grazzini, The structure of large logarithmic corrections at small transverse momentum in hadronic collisions, Nucl. Phys. B 616 (2001) 247 [hep-ph/0108273] [INSPIRE].

[80] S. Catani, The singular behavior of QCD amplitudes at two loop order, Phys. Lett. B 427 (1998) 161 [hep-ph/9802439] [INSPIRE].

[81] G.F. Sterman and M.E. Tejeda-Yeomans, Multiloop amplitudes and resummation, Phys. Lett. B 552 (2003) 48 [hep-ph/0210130] [INSPIRE].

[82] T. Becher and M. Neubert, Infrared singularities of scattering amplitudes in perturbative QCD, Phys. Rev. Lett. 102 (2009) 162001 [Erratum ibid. 111 (2013) 199905] [arXiv: 0901.0722] [INSPIRE].

[83] E. Gardi and L. Magnea, Factorization constraints for soft anomalous dimensions in QCD scattering amplitudes, JHEP 03 (2009) 079 [arXiv:0901.1091] [INSPIRE].

[84] O. Almelid, C. Duhr and E. Gardi, Three-loop corrections to the soft anomalous dimension in multileg scattering, Phys. Rev. Lett. 117 (2016) 172002 [arXiv:1507.00047] [INSPIRE].

[85] O. Almelid, C. Duhr, E. Gardi, A. McLeod and C.D. White, Bootstrapping the QCD soft anomalous dimension, JHEP 09 (2017) 073 [arXiv:1706.10162] [INSPIRE].

[86] A. Bassetto, M. Ciafaloni and G. Marchesini, Jet structure and infrared sensitive quantities in perturbative QCD, Phys. Rept. 100 (1983) 201 [INSPIRE].

[87] S. Catani, New techniques for calculating higher-order QCD corrections, in Proceedings, workshop on new techniques for calculating higher order $Q C D$ corrections, Zurich, Switzerland, 16-18 December 1992. 